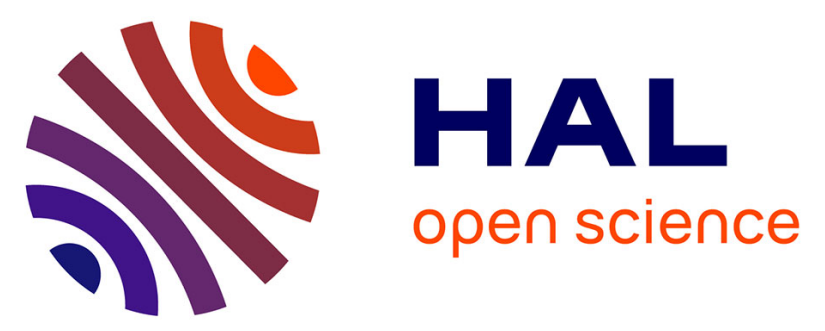

\title{
The Physcomitrium (Physcomitrella) patens PpKAI2L receptors for strigolactones and related compounds function via MAX2-dependent and independent pathways
}

Mauricio Lopez-Obando, Ambre Guillory, François-Didier Boyer, David Cornu, Beate Hoffmann, Philippe Le Bris, Jean-Bernard Pouvreau, Philippe Delavault, Catherine Rameau, Alexandre de Saint Germain, et al.

\section{- To cite this version:}

Mauricio Lopez-Obando, Ambre Guillory, François-Didier Boyer, David Cornu, Beate Hoffmann, et al.. The Physcomitrium (Physcomitrella) patens PpKAI2L receptors for strigolactones and related compounds function via MAX2-dependent and independent pathways. The Plant cell, 2021, 33, pp.3487-3512. 10.1093/plcell/koab217 . hal-03366148

\author{
HAL Id: hal-03366148 \\ https://hal.science/hal-03366148
}

Submitted on 5 Oct 2021

HAL is a multi-disciplinary open access archive for the deposit and dissemination of scientific research documents, whether they are published or not. The documents may come from teaching and research institutions in France or abroad, or from public or private research centers.
L'archive ouverte pluridisciplinaire HAL, est destinée au dépôt et à la diffusion de documents scientifiques de niveau recherche, publiés ou non, émanant des établissements d'enseignement et de recherche français ou étrangers, des laboratoires publics ou privés. 


\section{RESEARCH ARTICLE}

The Physcomitrium (Physcomitrella) patens PpKAI2L receptors for strigolactones and related compounds function via MAX2-dependent and independent pathways

Mauricio Lopez-Obando ${ }^{1,2,3,7}$, Ambre Guillory ${ }^{1,7}$, François-Didier Boyer ${ }^{4}$, David Cornu ${ }^{5}$,
Beate Hoffmann ${ }^{1}$, Philippe Le Bris ${ }^{1}$, Jean-Bernard Pouvreau ${ }^{6}$, Philippe Delavault ${ }^{6}$,
Catherine Rameau ${ }^{1}$, Alexandre de Saint Germain ${ }^{1 *}$, Sandrine Bonhomme ${ }^{1 *}$

${ }^{1}$ Institut Jean-Pierre Bourgin, INRAE, AgroParisTech, Université Paris-Saclay, 78000 Versailles, France

${ }^{2}$ Department of Plant Biology, Swedish University of Agricultural Sciences, The Linnean Centre for Plant Biology in Uppsala, SE-750 07, Uppsala, Sweden

${ }^{3}$ VEDAS Corporación de Investigación e Innovación (VEDASCII), Cl 8 B 65-261050024, Medellín, Colombia

${ }^{4}$ Université Paris-Saclay, CNRS, Institut de Chimie des Substances Naturelles, UPR 2301, 91198, Gif-sur-Yvette, France

${ }^{5}$ Université Paris-Saclay, CEA, CNRS, Institute for Integrative Biology of the Cell (I2BC), 91198, Gif-sur-Yvette, France

${ }^{6}$ Université de Nantes, Laboratoire de Biologie et Pathologie Végétales, LBPV, EA1157, 44000, Nantes, France

${ }^{7}$ These authors contributed equally to this work

*Corresponding authors: Sandrine.Bonhomme@inrae.fr and Alexandre.De-SaintGermain@inrae.fr

Short title: Defining strigolactone receptors in P. patens

One-sentence summary: The study of moss PpKAI2L receptors for strigolactones and related compounds highlights MORE AXILLARY GROWTH2-dependent and independent pathways for the perception of these compounds.

The authors responsible for distribution of materials integral to the findings presented in this article in accordance with the policy described in the Instructions for Authors (www.plantcell.org) are Sandrine Bonhomme (Sandrine.Bonhomme@inrae.fr) and Alexandre de Saint Germain (Alexandre.De-Saint-Germain@inrae.fr).

\section{ABSTRACT}

In angiosperms, the $\alpha / \beta$ hydrolase DWARF14 (D14), along with the F-box protein MORE AXILLARY GROWTH2 (MAX2), perceives strigolactones (SL) to regulate developmental processes. The key SL biosynthetic enzyme CAROTENOID CLEAVAGE DIOXYGENASE8 (CCD8) is present in the moss Physcomitrium patens, and PpCCD8-derived compounds regulate moss extension. The PpMAX2 homolog is not involved in the SL response, but 13 
PpKAI2LIKE (PpKAI2L) genes homologous to the D14 ancestral paralog KARRIKIN INSENSITIVE2 (KAI2) encode candidate SL receptors. In Arabidopsis thaliana, AtKAI2 perceives karrikins and the elusive endogenous KAI2-Ligand (KL). Here, germination assays of the parasitic plant Phelipanche ramosa suggested that PpCCD8-derived compounds are likely non-canonical SLs. (+)-GR24 SL analog is a good mimic for PpCCD8-derived compounds in $P$. patens, while the effects of its enantiomer (-)-GR24, a KL mimic in angiosperms, are minimal. Interaction and binding assays of seven PpKAI2L proteins pointed to the stereoselectivity towards (-)-GR24 for a single clade of PpKAI2L (eu-KAI2). Enzyme assays highlighted the peculiar behavior of PpKAI2L-H. Phenotypic characterization of Ppkai2l mutants showed that eu-KAI2 genes are not involved in the perception of PpCCD8derived compounds but act in a PpMAX2-dependent pathway. By contrast, mutations in $P p K A I 2 L-G$, and $-J$ genes abolished the response to the (+)-GR24 enantiomer, suggesting that PpKAI2L-G, and -J proteins are receptors for moss SLs.

\section{INTRODUCTION}

2 Strigolactones (SLs) are butenolide compounds with dual roles in plants: exuded in soil, SLs 3 signal the presence of a host to arbuscular mycorrhizal (AM) fungi (Akiyama et al. 2005; Besserer et al. 2006) and thus favor the establishment of symbiosis; as endogenous compouds, they (or derived compounds) play a hormonal role in developmental programs (Gomez-Roldan et al. 2008; Umehara et al. 2008) for reviews: (Lopez-Obando et al. 2015; Waters et al. 2017). SLs exuded from plant roots also act as signalling molecules in the rhizosphere, inducing parasitic plant seed germination (Cook et al. 1966) for review: (Delavault et al. 2017). SLs have been found in most land plants, including bryophytes, lycophytes, gymnosperms, and angiosperms (Yoneyama et al. 2018b). However, their synthesis and signaling pathways have mainly been described in angiosperms where core enzyme pathways involving DWARF27 (D27) isomerase and two CAROTENOID CLEAVAGE DIOXYGENASEs (CCD7 and CCD8) convert carotenoids into carlactone (CL). To date, CL is the reported precursor of all known SLs (Alder et al. 2012) and the substrate for further enzymes such as the cytochrome-P450 MORE AXILLARY GROWTH1 (MAX1) (for review: (Al-Babili and Bouwmeester 2015). Depending on the plant species, $\mathrm{CL}$ is converted into canonical or non-canonical SLs. These differ in the structure attached to the conserved enol ether-D ring moiety, which is shared by all SLs and essential for biological activity (Yoneyama et al. 2018a; Yoneyama 2020). In angiosperms, SLs are perceived by the $\alpha / \beta$ hydrolase DWARF14 (D14)/DECREASED APICAL DOMINANCE2 (DAD2)/RAMOSUS 3 (RMS3) (Arite et al. 2009; Hamiaux et al. 2012; de Saint Germain et al. 2016), which interacts with the F-box protein MORE AXILLARY GROWTH2 (MAX2) to target SUPPRESSOR OF MAX2-LIKE (SMXL) repressor proteins for proteasome degradation (Soundappan et al. 2015; Waters et al. 2015a). An unusual aspect 
of SL perception is that the D14 protein is both a receptor and an enzyme that cleaves its substrate (and covalently binds part of the SL) in a signaling mechanism that is still under debate (Yao et al. 2016; de Saint Germain et al. 2016; Shabek et al. 2018; Seto et al. 2019). In all cases, the pocket of the $\alpha / \beta$ hydrolase appears to be essential for substrate/ligand (SL) interactions (for review: (Bürger and Chory 2020).

The evolutionary origins of SLs, and in particular whether their primary role is that of a hormone or rhizospheric signal, are still unclear. The identification and quantification of SLs are a challenge in many species due to the very low amounts of the molecules present in plant tissues or exudates and their high structural diversification (Xie 2016; Yoneyama et al. 2018b). Therefore, the occurrence of SLs in a species was often inferred from the presence of the core biosynthesis enzymes encoded in its genome (Delaux et al. 2012; Walker et al. 2019) or from germination assays using plant exudates on parasitic plant seeds (Yoneyama et al. 2018b). Recently, SLs were proposed to only be produced in land plants (Walker et al. 2019). Evidence of a signaling pathway ancestral to the SL pathway came from the identification of an ancient D14 paralog named KARRIKIN INSENSITIVE2/HYPOSENSITIVE TO LIGHT (KAI2/HTL) during a screening of Arabidopsis thaliana mutants (Waters et al. 2012). Like D14, KAI2 is also an $\alpha / \beta$ hydrolase that interacts with the MAX2 F-box protein in a pathway regulating Arabidopsis seed germination and seedling development (Nelson et al. 2011; Waters et al. 2012). KAI2 is also involved in stress tolerance, drought tolerance, and AM symbiosis (Gutjahr et al. 2015; Wang et al. 2018; Villaecija-Aguilar et al. 2019; Li et al. 2020). However, the endogenous signal perceived by KAI2 remains unknown and is reported thus far as the KAI2Ligand (KL) (Conn and Nelson 2015).

To gain insight into SL signaling evolution, we focused our studies on a model for non-vascular plants, Physcomitrium (Physcomitrella) patens ( $P$. patens). Along with hornworts and liverworts, mosses such as P. patens belong to the bryophytes (Bowman et al. 2019). Bryophytes are currently described as a monophyletic group of embryophytes sharing an ancestor with vascular plants (Puttick et al. 2018; Harris et al. 2020). Therefore, comparing signaling pathways between extant vascular plants and extant bryophytes can provide insights into the evolutionary origin of these pathways (Guillory and Bonhomme 2021b). Furthermore, studying extant bryophytes may provide clues for understanding how the first plants have been able to survive out of water, and conquer land, 450 million years ago (Bowman et al. 2019; Blázquez et al. 2020; Harris et al. 2020). 
In P. patens, several D27 homologs, as well as both CCD enzymes (PpCCD7 and PpCCD8) required for SL synthesis are found (Proust et al. 2011), and CL has been detected as the product of PpCCD8 (Decker et al. 2017). The extended phenotype of Ppccd8 mutant plants indicates that PpCCD8-derived molecules are required for regulating moss filament growth. These molecules also act as a growth-limiting signal between neighboring moss plants, as they are exuded into the medium (Proust et al. 2011). PpCCD8-derived molecules also appear to play a role in rhizoid elongation and gametophore shoot branching (Delaux et al. 2012; Coudert et al. 2015). Application of the artificial SL ( \pm )-GR24 complemented the Ppccd8 mutant phenotype, suggesting that PpCCD8-derived molecules are indeed SL-like compounds (Proust et al. 2011). However, the exact nature of PpCCD8-derived molecules is still elusive (Yoneyama et al. 2018b), and the absence of $M A X 1$ homologs in P. patens suggests that the biosynthesis pathway in this moss may differ from that of vascular plants. Nevertheless, phylogenetic analysis of MAX1 homologs highlights the presence of this gene in other mosses and suggests that the biosynthesis pathway is otherwise conserved in land plants (Walker et al. 2019).

SL signaling also seems to differ between angiosperms and $P$. patens. Indeed, contrary to its angiosperm homolog, PpMAX2 is likely not involved in the response to PpCCD8-derived molecules, as the corresponding mutant does respond to ( \pm )-GR24 (Lopez-Obando et al. 2018). The PpMAX2 F-box protein appears to be involved in a light-dependent pathway required for early moss development and the regulation of gametophore number and size. No true homolog for the D14 SL receptor is found in the P. patens genome, whereas between 11 and 13 PpKAI2LIKE (PpKAI2L) candidate genes were described, depending on which version of the P. patens genome was searched. These genes, first called PpD14La to PpD14Lk (Delaux et al. 2012), were renamed PpKAI2L-A to PpKAI2L-M (Lopez-Obando et al. 2016a) and grouped into four subclades. Hereafter, for simplicity, we renamed the subclades as eu-KAI2 (previous clade (i), including PpKAI2L-A to -E); (FK), including PpKAI2L-F and -K (previous clade (ii)); (HIL), including PpKAI2L-H, -I, -L (previous clade (i.i-i.ii); (GJM) including PpKAI2L-G, -J, and M (previous clade iii). A comprehensive phylogenetic assessment placed the $P$. patens clades (FK), (HIL), and (GJM) into a super clade called DDK (D14/DLK2/KAI2) containing spermatophyte (angiosperm and gymnosperm) D14 clades, while the eu-KAI2 clade is highly conserved and common to all land plants (Bythell-Douglas et al. 2017). Nevertheless, moss proteins from the DDK clade were found to be as different from D14 as from KAI2 (BythellDouglas et al. 2017). 
89 Prediction of PpKAI2L protein structures found various pocket sizes, as observed for D14 and

90 KAI2 from vascular plants (Lopez-Obando et al. 2016a). Larger pocket sizes were predicted 91 for PpKAI2L-F and -K, while smaller pockets were predicted for eu-KAI2 clade proteins. 92 Consequently, these proteins could be receptors with diverse substrate preferences and might bind to either PpCCD8-derived compounds or the elusive KL. Accordingly, in our previous study of PpMAX2, we hypothesized that this F-box protein might be involved in a putative $P$. patens KL signaling pathway (Lopez-Obando et al. 2018). However, the involvement of PpKAI2L proteins in the PpMAX2 pathway remains an open question. The crystal structures of PpKAI2L-C, -E, and -H were recently published (Bürger et al. 2019). In vitro purified PpKAI2L proteins -C, -D, and -E (eu-KAI2 clade) were destabilized by (-)-5-deoxystrigol, a canonical SL with non-natural stereochemistry, but the binding affinity for the pure enantiomer was not determined. By contrast, PpKAI2L proteins $-\mathrm{H}$, $-\mathrm{K}$, and $-\mathrm{L}$ could bind to the karrikin $\mathrm{KAR}_{1}$ (Bürger et al. 2019). Proteins from the (GJM) clade were not studied, and no evidence for a role of one (or several) PpKAI2L as receptors for PpCCD8-derived molecules was provided, nor were experiments carried out in P. patens to validate the results. Moreover, the involvement of PpKAI2L proteins in the putative PpMAX2-dependent KL signaling pathway remains to be explored.

The aim of the present study was to investigate the nature of the PpCCD8-derived molecules in moss and to identify the moss receptors for these compounds. In an attempt to shed light on the type of SLs derived from PpCCD8, we first tested the activity of $P$. patens as a stimulant for seed germination of the root parasitic plant Phelipanche ramosa ( $P$. ramosa), for which one SL receptor, PrKAI2d3, has been recently characterized (de Saint Germain et al. 2021b). We then

112 looked for mimics of SL and KL that we could use in assays on P. patens. So far, the racemic

$113( \pm)$-GR24 has been used as a SL analog, but recent reports in angiosperms found that the 114 different enantiomers present in this synthetic mixture do not have the same effect (Scaffidi et 115 al. 2014). Indeed, (+)-GR24, (also called GR24 $4^{5 \mathrm{DS}}$ ) with a configuration close to the natural 116 strigol is mostly perceived by D14 and mimics CCD8-derived SLs (e.g. CL). On the other hand, 117 as for (-)-5-deoxystrigol, the configuration of (-)-GR24 (also called GR24 ${ }^{\text {ent-5DS}}{ }^{\text {) has so far not }}$ 118 been found in natural SLs. However, KAI2 perceives the (-)-GR24 analog better than D14 119 proteins, and (-)-GR24 has therefore been described as a KL mimic (Scaffidi et al. 2014; Zheng 120 et al. 2020). We tested both (+)-GR24 and (-)-GR24 isomers in phenotypic assays. Then, 121 refining and supplementing the work of (Bürger et al. 2019), we fully characterized seven 
122 PpKAI2L proteins in vitro by testing their cleavage activity and binding to pure GR24 123 enantiomers. We showed that stereoselectivity of most of the PpKAI2L proteins for GR24 124 enantiomers is weak, except for the eu-KAI2 clade, which shows preferential affinity for (-)-

125 GR24. We highlighted the stronger (compared to the other PpKAI2L) and non-selective 126 enzyme activity of PpKAI2L-H. We expressed these proteins in the Arabidopsis $d 14-1$ kai2-2

127 double mutant to examine conservation of the SL and/or KL perception function. Finally, we 128 used CRISPR-Cas9 technology to generate several $P$. patens multiple mutants affected in all 129 four PpKAI2L clades. By coupling analysis of these mutants' phenotypes and responses to pure 130 GR24 enantiomers with our biochemistry results, we provide strong evidence that eu-KAI2 131 clade PpKAI2L proteins could be moss KL receptors that function in a PpMAX2-dependent 132 pathway, while (GJM) clade PpKAI2L proteins would function as moss SL receptors in a 133 PpMAX2-independent pathway.

RESULTS

PpCCD8-derived compounds induce the germination of a hemp-specific population of Phelipanche ramosa

138 A recent report (Yoneyama et al. 2018b) indicated that canonical SLs previously identified in 139 P. patens tissues (Proust et al. 2011) could be contaminants. However other evidence suggests 140 that $P$. patens does synthesize SL-like products derived from CL. Indeed, PpCCD8 shows carlactone synthase activity (Decker et al. 2017), and both the synthetic SL analog ( \pm )-GR24 and CL do complement the Ppccd8 phenotype (Proust et al. 2011; Decker et al. 2017). Still,

143 quantification of SL and related compounds is a challenge in many species (Boutet-Mercey et 144 al. 2018; Yoneyama et al. 2018b; Rial et al. 2019; Floková et al. 2020), and so far no known 145 SL has been identified from P. patens (Yoneyama et al. 2018b). Here we tested the ability of $146 P$. patens exudates to induce the germination of parasitic seeds. Parasitic plants such as 147 Phelipanche ramosa can parasitize various host plants in response to specific exuded 148 germination stimulants (GS). Different genetic groups of $P$. ramosa seeds have been identified, 149 depending on the crop grown in the field where the seeds were collected (Huet et al. 2020). 150 Seeds from two populations of P. ramosa harvested in hemp (Cannabis sativa) ( $P$. ramosa 151 group 2a) and oilseed rape (Brassica napus) (P. ramosa group 1) fields (Stojanova et al. 2019; 152 Huet et al. 2020) were assayed with WT moss exudates (Figure 1). As a control, both groups 153 of seeds were germinated in the presence of $( \pm$ )-GR24 (Figure 1A). WT moss exudates induced 
154 the germination of $P$. ramosa group 2a seeds but not $P$. ramosa group 1 seeds (Figure 1A). In

155 another assay, $P$. ramosa seeds were added to culture plates close to WT or Ppccd8 plants, with 156 and without ( \pm )-GR24 (Figure 1B-C). P. ramosa group 2a but not group1 seeds germinated on

157 WT moss plates, while no germination was observed in the vicinity of Ppccd8 plants. In all 158 cases (WT and Ppccd8), the addition of ( \pm )-GR24 to the medium restored seed germination.

159 Thus, PpCCD8-derived compounds induce the germination of a specific population of $P$. 160 ramosa seeds, responding to not yet identified GS exuded by hemp.

$P$. patens responds strongly to (+)-GR24 and carlactone application but poorly to (-)GR24 and $\mathrm{KAR}_{2}$ in the dark

164 With the aim of determining which molecules could be used to mimic the yet unknown 165 PpCCD8-derived compounds and moss KL, we tested both enantiomers of GR24 ((+)-GR24 and (-)-GR24), the SL precursor CL as racemic form, and the karrikin $\mathrm{KAR}_{2}$. To quantify the phenotypic response of $P$. patens to SL, we counted the number of caulonemal filaments per plant grown in the dark (Guillory and Bonhomme 2021a) (Figure 2). The number of caulonema filaments decreased following the application of (+)-GR24 in WT and Ppccd8 in a dosedependent manner (Figure 2A-B). A dose of $0.1 \mu \mathrm{M}$ was sufficient to observe a clear and significant response in both genotypes (Figure 2). No significant changes in caulonema filament number were observed with (-)-GR24, except in WT, for which the $0.1 \mu \mathrm{M}$ and $10 \mu \mathrm{M}$ doses led to a significant increase (Figure 2A). However, in further assays (see below, Figure 9), this effect of (-)-GR24 was not repeatable.

Recent biochemistry experiments (Bürger et al. 2019) showed that some PpKAI2L proteins could bind to $\mathrm{KAR}_{1}(\mathrm{PpKAI} 2 \mathrm{~L}-\mathrm{H}, \mathrm{K}$ and $\mathrm{L}$ ), while a previous study concluded that $P$. patens was insensitive to $\mathrm{KAR}_{1}$ (Hoffmann et al. 2014). In the present work, we tested the $\mathrm{KAR}_{2}$ molecule, described as being more active than $\mathrm{KAR}_{1}$ in Arabidopsis (Waters et al. 2015a; Yao et al. 2021) (Figure 2A-B). $\mathrm{KAR}_{2}$ has an unmethylated butenolide group, unlike $\mathrm{KAR}_{1}$. In WT, no significant effect on caulonema number was observed following the application of

182 increasing doses of $\mathrm{KAR}_{2}$ (Figure 2A). In Ppccd8, we observed an increase in filament number at $10 \mu \mathrm{M}$ (Figure 2B). To conclude, the phenotypic effects of $\mathrm{KAR}_{2}$ on P. patens were slight and not clearly dose responsive. 
186 We also tested CL, described as the natural product of PpCCD8 in P. patens (Decker et al. 187 2017). As previously reported (Decker et al. 2017), racemic CL application had a negative 188 effect on caulonema filament number for both WT and Ppccd8 plants; however, in our assays, the effect was only significant at $10 \mu \mathrm{M}$ for both genotypes (Figure $2 \mathrm{C}$ ).

Overall, findings from these phenotypic assays suggest that GR24 enantiomers have distinct effects in P. patens, as observed in Arabidopsis (Scaffidi et al. 2014). Indeed, the (+)-GR24 analog mimics the effects of CL, although it is far more potent, and can thus be used to mimic the effects of PpCCD8-derived compounds. On the other hand, the (-)-GR24 analog and $\mathrm{KAR}_{2}$ have slight phenotypic effects that are not always consistent or even tend to be opposite those of PpCCD8-derived compounds.

All $P$ PKAI2L genes are expressed at relatively low levels and putatively encode proteins with a conserved catalytic triad

Like D14 and KAI2 genes, all 13 PpKAI2L genes encode a catalytic triad (Ser, His, Asp) (Figure 3 and Supplemental Figure S1). To test if the high number of PpKAI2L genes hints at different spatial and temporal expression profiles, we obtained the expression patterns of all $P p K A I 2 L$ genes in $P$. patens, using a cDNA library from various organs/tissues (Supplemental Figure S2). $P p K A I 2 L$ genes transcripts were detected in all tested tissues, at relatively low levels compared to the control genes. Quantitative RT-PCR could not be used to assess the expression of $P p K A I 2 L-M$, as its predicted transcript is almost identical to that of $P p K A I 2 L-G$, and the observed transcript levels are attributable to both $P p K A I 2 L-G$ and $-M$. In spores, $P p K A I 2 L-F$ and $-J$ had higher transcript levels than other PpKAI2L genes. In protonema and gametophores, however, PpKAI2L-D from eu-KAI2 clade showed the highest transcript levels among PpKAI2L genes (Supplemental Figure S2A). The data are consistent with those previously reported (Ortiz-Ramirez et al. 2016; Perroud et al. 2018).

The PpKAI2L-C, -D, and -E proteins are destabilized by (-)-GR24, as observed for AtKAI2; PpKAI2L-F, -K, -L, and -H interact weakly with GR24 enantiomers

To investigate whether the PpKAI2L proteins behave similarly to AtD14 or AtKAI2 in vitro, 216 we cloned the coding sequences (CDS) of all PpKAI2L genes and over-expressed them in $E$. 217 coli. After the successful purification and solubilization of seven PpKAI2L proteins (-C,-D,- 
218 E,-F,-H,-K and -L), we investigated their interactions with SL analogs and their potential 219 enzymatic activities. Unfortunately, due to low solubility, the six other PpKAI2L proteins could 220 not be purified in sufficient amounts to ensure their quality. We tested the interactions of the 221 purified PpKAI2L proteins with SL analogs via nano Differential Scanning Fluorimetry 222 (nanoDSF) (Figure 4 and Supplemental Figure S3). (+)-GR24 and (-)-GR24 enantiomers 223 destabilized AtD14 (Supplemental Figure S3A), while AtKAI2 was destabilized by (-)-GR24 224 addition only (Supplemental Figure S3B), as previously reported (Waters et al. 2015a). All 225 tested eu-KAI2 clade proteins (PpKAI2L-C -D and -E) were destabilized when (-)-GR24 was 226 added, as was AtKAI2 (Supplemental Figure S3C to 3E), in accordance with the reported 227 stereoselectivity for un-natural (-)-5DS (Bürger et al. 2019). Puzzlingly, the PpKAI2L-C, and 228 -E proteins showed a tendency to be stabilized by (+)-GR24 at high concentrations 229 (Supplemental Figure S3C-E), which was not reported when using (+)-5DS. PpKAI2L-F and 230 PpKAI2L-L also showed a slight increase in $\mathrm{T}_{\mathrm{m}}$ following the addition of both (-)-GR24 and $231(+)$-GR24 $\left(\leq 1^{\circ} \mathrm{C}\right)$, suggesting a slight stabilization (Figure 4 and Supplemental Figure S3F, 3I). 232 None of the GR24 isomers affected the stability of the PpKAI2L-K or PpKAI2L-H proteins 233 (Supplemental Figure S3G, 3H).

234 Since we sometimes observed opposite effects of different isomers on the stability of the 235 PpKAI2L proteins, we reasoned that their binding affinity had to be assessed for the GR24 236 isomers, rather than for ( \pm )-GR24, as previously reported (Bürger et al. 2019). Binding affinities 237 were quantified with $K_{\mathrm{d}}$ affinity calculations following intrinsic tryptophan fluorescence 238 measurements (Figure 4 and Supplemental Figure S4). The affinity for (+)-GR24 could be 239 evaluated for PpKAI2L-K and PpKAI2L-H only $\left(K_{\mathrm{d}}\right.$ superior to $\left.100 \mu \mathrm{M}\right)$, and was weaker than 240 that of AtD14 $(23 \mu \mathrm{M})$. No $K_{\mathrm{d}}$ value for $(+)$-GR24 could be determined for AtKAI2, eu-KAI2, 241 or PpKAI2L-F, suggesting a very weak affinity for (+)-GR24 or a lack of binding. With (-)242 GR24, comparable affinities were found for AtKAI2 $(45 \mu \mathrm{M})$, AtD14 (94 $\mu \mathrm{M})$, PpKAI2L-D $243(92 \mu \mathrm{M})$, PpKAI2L-E $(39 \mu \mathrm{M})$, and PpKAI2L-K $(41 \mu \mathrm{M})$. A much weaker affinity for $(-)$ 244 GR24 was found for PpKAI2L-H $(273 \mu \mathrm{M})$.

246 PpKAI2L-C, -D, -E, -K, and -L preferentially cleave (-)-GR24, while PpKAI2L-H cleaves 247 both (-)-GR24 and (+)-GR24 
As all PpKAI2L proteins contain the conserved catalytic triad, and since we found that most of them were able to bind to at least one of the GR24 isomers, we tested their enzyme activity against SL analogs. First, when incubated with the generic substrate for esterases, 4-nitrophenyl acetate ( $p$-NPA), AtKAI2 and all tested PpKAI2L proteins showed enzyme activity (Supplemental Figure S5A-B), consistent with a previous report (Bürger et al. 2019). Kinetic constants were in the same range for all proteins but one, and similar to that of AtKAI2. The exception was PpKAI2L-H, which had a higher $V_{\max }$ and $K_{M}$, highlighting faster catalysis and a better affinity for $p$-NPA than all the others (Supplemental Figure S5C). We further characterized the enzyme activity of PpKAI2L proteins on the GR24 isomers. We compared their substrate bias to that of the pea (Pisum sativum) SL receptor RMS3/PsD14 and to that of AtKAI2 (Figure 4 and Supplemental Figure S6). None of the PpKAI2L proteins showed as high an enzyme activity as RMS3/PsD14 (100\% cleavage of (+)-GR24 and (-)-GR24 (de Saint Germain et al. 2016)). Only PpKAI2L-H showed a relatively high catalytic activity towards the GR24 isomers, especially towards (-)-GR24 (almost 70\%, Supplemental Figure S6). AtKAI2 and all other tested PpKAI2L proteins except one selectively cleaved the (-)-GR24 enantiomer, but with low activity (less than 28\%, observed for AtKAI2). Finally, PpKAI2L-F showed very low enzyme activity towards both isomers (less than 5\%).

The higher cleavage activity of PpKAI2L-H on synthetic SL analogs is explained by the presence of a specific leucine residue

The higher enzyme activity of PpKAI2L-H (Supplemental Figure S6) and its lack of thermal shift when incubated with GR24 isomers (Supplemental Figure S3H) set this protein apart from other PpKAI2L proteins. To better characterize PpKAI2L-H enzyme activity, we used a profluorescent probe as substrate $(( \pm)-G C 242)$, in which the ABC rings of GR24 are replaced by a coumarine-derived moiety (DiFMU) (de Saint Germain et al. 2016). ( \pm )-GC242 is bioactive on moss, as it reduced the number of caulonema filaments in the dark in a dose-responsive manner (evaluated on the Ppccd8 mutant, Supplemental Figure S7A). The use of ( \pm )-GC242 as a substrate confirmed the relatively high enzyme activity of PpKAI2L-H compared to all the other PpKAI2L proteins (Supplemental Figure S7B). Indeed, after 2 hours, PpKAI2L-H catalyzed the formation of $1 \mu \mathrm{M}$ DiFMU, while other PpKAI2L activities were indistinguishable from background noise. However, the PpKAI2L-H enzymatic profile did not show the biphasic curve (a short burst phase, quickly followed by a plateau phase), which 
characterizes the single turnover activity of AtD14 (Supplemental Figure S7B (de Saint

281 Germain et al. 2016)). The lack of a plateau for PpKAI2L-H suggested that this protein acted as a Michaelian enzyme on the SL analog.

283 To try to understand this singularity, we compared the solvent-exposed residues in the binding 284 pocket of the PpKAI2L proteins and noticed that PpKAI2L-H harbors a leucine ${ }^{28}\left(\mathrm{Leu}^{28}\right)$ 285 residue instead of the phenylalanine found in AtD14 $\left(\mathrm{Phe}^{26}\right)$, AtKAI2, and all other PpKAI2L 286 proteins (Figure 5A). The Phe residue is located at the junction between helix $\alpha 4$ and $\alpha 5$, near 287 the catalytic site (Figure 5B), and the crystal structure of PpKAI2L-H (Bürger et al. 2019) 288 indicates that this residue interacts with the D-ring of the SL. Furthermore, a mutant PpKAI2L$289 \mathrm{H}$ protein where $\mathrm{Leu}^{28}$ is changed to Phe showed a single turnover profile similar to AtD14, 290 both reaching a plateau at $0.4 \mu \mathrm{M}$ DiFMU, correlating with the protein concentration (Figure 291 5C and 5D). PpKAI2L-H and PpKAI2L-H ${ }^{\text {Leu28Phe }}$ proteins had comparable affinity towards $( \pm)$ $292 \operatorname{GC} 242\left(K_{1 / 2}=4,794 \mu \mathrm{M} v s 4,675 \mu \mathrm{M}\right)$ but showed different $V_{\max }$ values $\left(V_{\max }=0,06794 \mu \mathrm{M} \min ^{-}\right.$ $293{ }^{1}$ vs $0,01465 \mu \mathrm{M} \cdot \mathrm{min}^{-1}$ ), suggesting that the $\mathrm{Leu}^{28}$ residue affects the velocity of catalytic 294 activity (Figure 5C and 5D).

295

Moss PpKAI2L proteins, like vascular plant receptors, covalently link GR24 enantiomers

297 To further investigate whether PpKAI2L proteins play roles as receptors of SLs and related 298 compounds, we examined the covalent attachment of the GR24 isomers to the PpKAI2L 299 proteins (Figure 4 and Supplemental Figure S8). Mass spectrometry analyses revealed 96 Da 300 increments (corresponding to the D ring mass) when AtKAI2 and PpKAI2L-C, -D, -E, -F, or -

301 L were incubated with (-)-GR24. Strikingly, 96 Da increments were also observed when 302 PpKAI2L-E, -F, -L, and -K were incubated with the other isomer (+)-GR24, in contrast to other 303 reports (Bürger et al. 2019). However, for PpKAI2L-E, the intensity peak was much lower with 304 (+)-GR24 than with (-)-GR24, confirming the better affinity for the latter (Figure 4 and 305 Supplemental Figure S8). PpKAI2L-H did not covalently bind the D ring following incubation 306 with either enantiomer, further suggesting that it displays Michaelian enzyme activity.

307 Thus, poor interactions were observed with (+)-GR24, which was reported to mimick SLs and 308 had the most potent effect on P. patens in our phenotypic assays (Figure 2). Strikingly, all eu309 KAI2 clade proteins tested showed the strongest affinity for the (-)-GR24 enantiomer (Figure 310 4), which is reported to be a good mimic for the putative KL in vascular plants (Scaffidi et al. 
2014; Zheng et al. 2020). We then carried out in planta studies to investigate if these PpKAI2L

312 homologs are necessary for SL or KL perception.

313

314 None of the PpKAI2L genes complement the Arabidopsis d14-1 kai2-2 double mutant

316 We used cross species complementation assays to test whether any of the PpKAI2L proteins

317 could carry out similar functions in SL and/or KL signaling to that of AtD14 and/or AtKAI2 in

318 Arabidopsis (Figure 6). CDS of the PpKAI2L-C, $-D,-F,-G$, and $-J$ genes were cloned

319 downstream of the AtD14 or AtKAI2 promoter, and the resulting constructs were expressed in

320 the Arabidopsis double mutant Atd14-1 kai2-2, which shows both a hyperbranched phenotype

321 and elongated hypocotyls (Supplemental Figure S9). As controls, the double mutant was

322 transformed with the AtKAI2 or AtD14 CDS under the control of endogenous promoters. Only

323 lines expressing $A t D 14$ under the control of the $A t D 14$ promoter fully restored rosette branching

324 to WT (Ler) values (Figure 6A). Under the control of the AtD14 promoter, neither AtKAI2 nor

325 any of the PpKAI2L genes significantly restored the branching phenotype of Atd14-1 kai2-2.

326 We conclude that none of the tested PpKAI2L genes can fully complement the function of

327 AtD14 in shoot branching.

328

329 We also examined possible complementation of the AtKAI2 function in the Atd14-1 kai2-2

330 mutant by monitoring hypocotyl length under low light conditions, with or without $1 \mu \mathrm{M}(+)-$

331 GR24 or (-)-GR24 in the culture medium (Figure 6B and Supplemental Figure S9). Compared

332 to WT, the double mutant showed longer hypocotyls under control conditions, as did the single

333 kai2-2 mutant. The addition of (+)-GR24 or (-)-GR24 had no effect on the phenotype of the

334 double mutant (Figure 6B). By contrast, (+)-GR24 treatment led to shorter kai2-2 hypocotyls,

335 likely due to perception and transduction by the AtD14 protein, which is still active in the single

336 mutant. As expected from the loss of AtKAI2 function, (-)-GR24 addition had no effect on

337 kai2-2 hypocotyls. Surprisingly, expressing AtKAI2 under the control of the AtKAI2 promoter

338 did not fully restore the hypocotyl length of the double mutant under our control conditions,

339 but it did restore the response to (-)-GR24, as anticipated (Figure 6B).

340

341 Under control conditions, almost all double mutant lines expressing AtD14 or one PpKAI2L

342 protein showed long hypocotyls (Figure 6B and Supplemental Figure S9B). Even longer

343 hypocotyl phenotypes (compared to the double mutant) were found under control conditions 
344 for one line expressing PpKAI2L-C (\#16.2) and one line expressing PpKAI2L-D (\#1.4)

345 (Supplemental Figure S9B). This unexpected effect of the introduced $\alpha / \beta$ hydrolases will be 346 discussed below. By contrast, a line expressing PpKAI2L-G (\#7.1) had short hypocotyls under

347 control conditions, suggesting that the expressed protein had indeed complemented the AtKAI2

348 function (Figure 6B). In a separate assay, in addition to shorter hypocotyls, all three lines

349 expressing PpKAI2L-G in the double mutant background showed significantly larger

350 cotyledons, further hinting at the restoration of AtKAI2 function (Supplemental Figure S9C).

351

352 When either (+)-GR24 or (-)-GR24 was added (Figure 6B), short hypocotyls (similar to WT) 353 were observed in the pKAI2:AtD14 expressing line, indicating that AtD14-mediated signal 354 transduction of both enantiomers occurs when AtD14 is present in tissues where AtKAI2 is 355 normally active. However, adding GR24 enantiomers to the medium had no such effect on most 356 lines expressing PpKAI2L proteins (Figure 6B and Supplemental Figure S9B). Overall, these 357 assays showed that PpKAI2L-G may mediate KL signaling in Arabidopsis hypocotyls, even 358 though it could not fully ensure the function of AtKAI2 in this process.

359

360 Multiplex editing of $P$ PKAI2L genes

361 Multiplex gene editing using CRISPR-Cas9 allowed us to isolate $P$. patens mutants affected in 362 one or several PpKAI2L genes (Lopez-Obando et al. 2016b) (Figure 3 and Supplemental Figure $363 \mathrm{~S} 10)$. For the eu-KAI2 clade, two triple $(a b c, c d e)$ and two quintuple mutants (abcde) were 364 chosen for further analysis (Supplemental Table S1). The remaining (HIL), (FK), and (GJM) 365 clades were targeted in separate experiments using combinations of specific CRISPR RNAs 366 (crRNAs) for each gene. As biochemistry experiments suggested a purely enzymatic role for 367 PpKAI2L-H, a single deletion mutant was obtained for the PpKAI2L-H gene through 368 homologous recombination, where the full CDS was removed from the moss genome $(\Delta h$ 369 mutant, Supplemental Figure S10 and S11). This $\Delta h$ mutant was employed in further 370 transformation experiments with crRNAs from the same (HIL) clade and/or from the (FK) and 371 (GJM) clades, generating the $\Delta$ hil and $\Delta h f k j$ mutants (Supplemental Table S1). Eventually, a $3727 \mathrm{X}$ mutant was obtained ( $\Delta$ hifkgjm) where all the mutations except those in PpKAI2L-J and $-M$ 373 were null (Supplemental Table S1 and see below). For convenience, null mutations are hereafter 374 indicated in bold letters. 

in other clades are more similar to WT or Ppccd8

378 Our rationale was that a mutant affected in the response to PpCCD8-derived compounds should 379 show a phenotype similar to that of the SL biosynthesis mutant Ppccd8. We first performed a 380 phenotypic analysis of the mutants in the light. After four weeks of culture, Ppccd8 plants were 381 slightly bigger than WT ((Proust et al. 2011), Figure 7), whereas Ppmax2-1 plants were smaller, 382 with fewer but bigger gametophores ((Lopez-Obando et al. 2018), Figure 7). The diameter of 383 mutants in eu-KAI2 clade genes was significantly smaller than that of Ppccd8 and WT and 384 slightly larger than that of Ppmax2-1 (Figure 7A-B). The phenotype of the eu-KAI2 clade mutants, with early and large gametophores, resembled that of Ppmax2-1, although not as pronounced (Figure 7A). To the naked eye, three week-old plants from mutants in genes from the (FK), (GJM), and (HIL) clades were indistinguishable from WT (Figure 7A). After a month of growth, however, all mutants affecting genes from the (FK) and (GJM) clades showed a slightly larger diameter, intermediate between that of WT and Ppccd8 (Figure 7D). Mutants in the (HIL) clade such as $\Delta h, \Delta h i$ and $\Delta h i l$ were comparable to WT (Figure 7D). Thus, based on mutant phenotypes in the light, eu-KAI2 clade genes would be involved in a PpMAX2dependent pathway, while (FK) and (GJM) clade genes would instead be involved in the PpCCD8-derived SLs pathway. Genes from the (HIL) clade would not be involved in either pathway.

Like Ppmax2, eu-KAI2 clade quintuple mutants are affected in photomorphogenesis

Mutants in eu-KAI2 clade genes showed the typical phenotype of the Ppmax2-1 mutant in white light. We previously showed that the Ppmax2-1 mutant is affected in photomorphogenesis under red light (Lopez-Obando et al. 2018). After two months of growth under red light, Ppmax2-1 gametophores were much more elongated than WT gametophores, whereas Ppccd8 gametophores were shorter (Figure 8). Among eu-KAI2 mutants, gametophores of both triple mutants $\boldsymbol{a b c}$ and $\boldsymbol{c} \boldsymbol{d e}$ were a similar height to WT. Interestingly, the quintuple mutant (abcde1) showed significantly elongated gametophores, similar to Ppmax2-1 (Figure 8A and C). The other tested quintuple mutant (abcde-2) also had elongated gametophores under red light, intermediate between WT and Ppmax2-1 (Supplemental Figure S12). The weak phenotypes of 
clade, as knockout (KO) mutations for PpKAI2L-A, B, C, and/or D did not result in plants with gametophores as elongated as those of the Ppmax2-1 mutant.

409 Gametophores from mutants where genes from the (FK) and/or (GJM) clades were mutated 410 (fkj, $\Delta h \boldsymbol{f k j}-1$ and $\Delta h \boldsymbol{f k g} \mathbf{g} m$ ) were similar in height to WT, suggesting that genes from clade (FK) 411 and clade (GJM) have no role in photomorphogenesis in red light (Figure 8B-C and 412 Supplemental Figure S12). (HIL) clade triple mutant $\Delta$ hil showed shorter gametophores under 413 red light, similar to Ppccd8 (Figure 8A). By contrast, the gametophores of both the single $\Delta \boldsymbol{h}$ 414 and the double $\Delta \boldsymbol{h i}$ mutants were intermediate in height between WT and Ppccd8 (Figure 8A415 B). This suggests a specific role for the (HIL) clade genes, with an opposite impact on 416 gametophore development compared to that of the PpMAX2 pathway.

417 In conclusion, the phenotypes of the Ppkai2L mutants in red light allowed us to differentiate 418 the functions of the eu-KAI2 clade genes. These genes are likely to be involved in a PpMAX2419 dependent pathway related to photomorphogenesis, whereas genes from the three other clades 420 (DDK superclade) are more likely to play roles independent of PpMAX2.

\section{Mutants in the (GJM) clade no longer respond to (+)-GR24 application}

423

424

425

426

427

To determine which of the Ppkai2L mutants carry mutations in potential receptors for PpCCD8compounds (SL-related) or other (KL-related) compounds, we tested their phenotypic responses to GR24 enantiomers at $0.1 \mu \mathrm{M}$ (Figure 9). In the assays reported above on WT and Ppccd8 in the dark (Figure 2), (-)-GR24 appeared to be a poor KL mimic in moss. However, the stereospecificity of the PpKAI2L proteins for (-)-GR24 led us to pursue our assays with this enantiomer.

For the eu-KAI2 clade, under control conditions, all mutants showed an equivalent number of filaments to WT, except for the quintuple mutant. The latter tended to have fewer filaments under control conditions, another similar phenotype to Ppmax2-1 (Figure 9A and Supplemental Figure S13A). The addition of $0.1 \mu \mathrm{M}(-)$-GR24 had no significant effect on eu-KAI2 clade mutants or WT (Figure 9A). However, in this assay, both Ppccd8 and Ppmax2-1 showed a significant decrease in caulonema filament number in response to $0.1 \mu \mathrm{M}(-)-\mathrm{GR} 24$. In a separate experiment, a dose of $1 \mu \mathrm{M}$ of (-)-GR24 had opposite effects on WT and Ppccd8 filament number, with an increase and decrease, respectively (Supplemental Figure S13A, and Figure 2 above), but had no significant effect on the Ppmax2-1 mutant, although a tendency 
towards a decrease was observed. At this higher dose, the quintuple eu-KAI2 clade mutant showed a significant decrease in caulonema filament number, like Ppccd8, but in contrast to WT. Thus, similar to PpMAX2 loss of function, mutating eu-KAI2 genes did not abolish a response to the (-)-GR24 enantiomer. The application of (+)-GR24 had a significant negative effect on the number of filaments for the quintuple abcde mutant, like for WT and the Ppccd8 and Ppmax2-1 mutants (Figure 9B). Thus, mutating any of the eu-KAI2 clade genes did not hamper the response to (+)-GR24, and therefore it likely does not hamper the responses to PpCCD8-derived compounds.

We then tested the effects of GR24 enantiomers on Ppkai2L mutants from the three other clades: (FK), (GJM), and (HIL). Strikingly, under control conditions, like Ppccd8, all the mutants had more filaments than WT, except for mutants in the (HIL) clade, which were similar to WT (Figure 9C to 9E) or tended to have fewer filaments (Supplemental Figure S13). Both the single $\boldsymbol{j}$ mutant and the quintuple mutant gjmil showed a significant response to (-)-GR24 (fewer caulonema), as did Ppccd8 (Figure 9C and Supplemental Figure S13A-B). No clear response to (-)-GR24 was seen in mutants with $\mathrm{KO}$ mutations in PpKAI2L-F, $-K,-H,-G,-M$, $-I$ or $-L$, like for WT (Figure 9C and Supplemental Figure S13A-B). Finally, we examined the response to (+)-GR24 for mutants of the (FK), (GJM), and (HIL) clades (Figure 9D-E and Supplemental Figure S13C-D). The number of caulonema was clearly reduced in WT and Ppccd8 (as shown above, Figure 2), and in mutants carrying the $\Delta h$ mutation, alone or in combination with $\boldsymbol{f}, \boldsymbol{k}, \boldsymbol{i}$ or $\boldsymbol{l}$ null mutations. Thus, genes from the (FK) and (HIL) clades do not play a role in the response to (+)-GR24. However, the response to the (+)-GR24 enantiomer was abolished in all mutants where the PpKAI2L-J gene was knocked out (Figure 9D: $\boldsymbol{j}$ and gjm-1, and Supplemental Figure S13C: gjmil and $4 h f k j-3$ and 13D: gjm-3 and gjm-5). Interestingly, in the two lines where the $j$ mutation was not null but the $P p K A I 2 L-G$ gene was knocked out (7x 4 hifkgjm and $\boldsymbol{g}$ jm-4 mutants), the response to (+)-GR24 was also abolished (Supplemental Figure S13C-D). Thus, based on phenotypic assays of mutant caulonema, both the PpKAI2L-J and $-G$ genes appear to be necessary for the response to (+)-GR24 and are therefore the best candidates for receptors to PpCCD8-derived SLs.

To further test if PpKAI2L-J and -G could be receptors for PpCCD8-derived SLs, we measured the transcript levels of SL-responsive genes in the corresponding mutants (Figure 10). We previously reported that in WT and Ppccd8 plants, PpKUF1LA gene transcript abundance 
470 is enhanced in the dark (Lopez-Obando et al. 2018). We used this marker along with the 471 Pp3c6_15020 gene (encoding a putative histidine kinase) that was previously found to be 472 upregulated by $( \pm)$-GR24 (Supplemental Figure S14). Using GR24 enantiomers, we confirmed 473 that the transcript levels of both genes increased following $1 \mu \mathrm{M}(+)$-GR24 addition in WT and 474 Ppccd8, but not in Ppmax2-1. Strikingly, an increase in transcript levels following (-)-GR24 475 application was observed for both markers in Ppccd8, and for PpKUF1LA only in WT (Figure 476 10). In the quintuple eu-KAI2 mutant, the addition of (+)-GR24 but not (-)-GR24 increased 477 PpKUF1LA and Pp3c6_15020 transcript levels. By contrast, in the $\boldsymbol{j}$ mutant, the transcript 478 levels of both genes increased following (-)-GR24 addition and were slightly increased 479 (PpKUF1LA) or unchanged (Pp3c6_15020) in response to (+)-GR24 application. In the gim-2 480 mutant, the response marker transcript levels were unchanged following either (+)-GR24 or 481 (-)-GR24 addition. Thus, the transcriptional responses of the tested mutants confirm the notion 482 that eu-KAI2 genes are not involved in the response to (+)-GR24, while this response is 483 impaired in (GJM) clade mutants. Only the Ppccd8 and $\boldsymbol{j}$ mutants showed a clear and significant 484 transcriptional response (for both markers) to (-)-GR24 addition.

\section{DISCUSSION}

\section{Are PpCCD8-derived products non-canonical SLs?}

P. patens exudates were previously reported to induce the germination of Orobanche ramosa (old name for Phelipanche ramosa) seeds (Decker et al. 2017), but the origin of the tested population was not specified. Here, we further demonstrated that PpCCD8-derived products are germination stimulants of $P$. ramosa group 2a seeds that were harvested in a hemp field but do not induce the germination of $P$. ramosa group 1 seeds collected from an oilseed rape field. Differences in the suceptibility of root parasitic weeds can be attributed to the chemical nature of host plant exudates (Yoneyama et al. 2018b). Our results suggest that the PpCCD8-derived products share similarities with hemp secondary metabolites. So far, no known canonical SLs have been isolated from hemp (Huet et al. 2020). Since $P$. patens likely produces CL (Decker et al. 2017) but lacks a true $M A X 1$ homolog (Proust et al. 2011), we hypothesize that PpCCD8derived compounds correspond to non-canonical SLs derived from CL or hydroxyl CLs (Yoneyama 2020). Further supporting this hypothesis, we previously showed that GR5, a noncanonical SL analog, was as bioactive as ( \pm )-GR24 on $P$. patens caulonema length (Hoffmann et al. 2014). As mimics of SLs in the present study, we used the (+)- and (-)-GR24 artificial 
analogs that were available at the time of our study (see below). Of note, both isomers are active on $P$. ramosa group 1 and group 2a seeds. However, the (+)-GR24 isomer, which has a canonical SL structure, has similar germination stimulating activity to $( \pm)-G R 24$, while the (-)GR24 isomer is far less active (de Saint Germain et al. 2019). For further identification of PpCCD8-derived SLs in the future, non-canonical SL analogs such as the recently described methyl phenlactonoates (Jamil et al. 2020) may be more appropriate (see also below).

\section{Looking for the best mimic of SLs or KL}

The (-)-GR24 analog has a non-natural configuration that has never been found in plant exudates, contrary to the (+)-GR24 enantiomer, which bears similarity to 5-deoxystrigol ((+)5DS) and strigol-type canonical SLs (Scaffidi et al. 2014). In our bioassays of moss phenotypes, CL application decreased the number of caulonema of both WT and Ppccd8 at $10 \mu \mathrm{M}$. A similar (but much stronger) effect was observed with (+)-GR24, which we thus consider to be the best mimic of PpCCD8-derived compounds available so far. It is not surprising that (+)-GR24 is more potent than CL, as the assays were carried out in aqueous medium, and natural SLs were described as being far less stable than synthetic analogs in aqueous medium (Akiyama et al. 2010; Boyer et al. 2012). Moreover, we cannot exclude the possibility that CL needs to be transformed into a more bioactive non-canonical SL in planta to trigger the effects observed here. By contrast, the effects of (-)-GR24 are weak, not dose responsive, and sometimes contradictory in WT versus Ppccd8. Indeed, in several assays, we observed a significant increase in caulonema number in WT (Figure 2A, Supplemental Figure S13A) while this number consistently decreased in Ppccd8, mimicking the result of SL application (Figure 9, Supplemental Figure S13A-B).

Interestingly, we also observed an increase in caulonema number when testing $\mathrm{KAR}_{2}$, although this increase was only significant at $10 \mu \mathrm{M}$ for Ppccd8 (Figure 2B). So far we had not observed any effect of karrikins $\left(\mathrm{KAR}_{1}\right)$ on the phenotypes of $P$. patens (Hoffmann et al. 2014), and this is thus the first hint of a possible effect of some karrikins on moss, which needs to be confirmed. We hypothesize that the increase in caulonema filament number is the effect triggered by the as yet unidentified moss KL (see also below and Figure 11). It is puzzling, however, that the effect of $\mathrm{KAR}_{2}$ is more easily observed in Ppccd8 (thus in the absence of SLs) than in WT, while the same effect of (-)-GR24 (increasing of filament number) is only seen in WT. We propose (Figure 11 and see below) that (GJM) clade PpKAI2L proteins perceive (-)-GR24, 
which would explain the apparent contrary effects of this enantiomer. In the absence of endogenous SL (in the Ppccd8 mutant), (-)-GR24 would thus trigger the SL pathway. This could also explain the transcriptional response to (-)-GR24 observed in Ppccd8 (Figure 10). Thus, (-)-GR24 is not a very robust mimic of the as yet unidentified moss $\mathrm{KL}$, and $\mathrm{KAR}_{2}$ is likely not a potent KL mimic either. This conclusion also suggests that $P$. patens $\mathrm{KL}$ may be quite different from that of angiosperms. Recently, desmethyl butenolides were reported to be more potent and to function as specific ligands for KAI2, both in vitro and in plants, including the lycophyte Selaginella moellendorffii (S. moellendorffii) and the bryophyte Marchantia polymorpha (M. polymorpha) (Yao et al. 2021). Thus, testing desmethyl butenolides, and in particular the (-)-desmethyl GR24 enantiomer (dGR24 ${ }^{\text {ent5DS }}$ ) on P. patens plants and proteins is a priority in the search for a KL mimic.

\section{Biochemical characterization suggests that PpKAI2L proteins from the eu-KAI2 clade act as KL receptors}

Among the analogs examined, we observed the best binding of the (-)-GR24 enantiomer to AtKAI2 and all tested PpKAI2L proteins from the eu-KAI2 clade. This confirms a recent report (Bürger et al. 2019) of the preferential binding of (-)-5DS by PpKAI2L-C, -D, and -E. In addition, we found that the (+)-GR24 enantiomer interacts poorly with these PpKAI2L proteins, but not with AtKAI2, indicating less stringency for moss eu-KAI2 clade proteins. Still, this result suggests that eu-KAI2 proteins share a perception mechanism with AtKAI2, and furthermore, they may recognize KL-like compounds. The moss KL compound(s) may, however, differ from the angiosperm KL, since the expression of PpKAI2L-C or -D did not complement the kai2-2 hypocotyl phenotype in Arabidopsis, or more likely, the protein partners in Arabidopsis are different (see also below).

It should be noted that AtKAI2 is degraded following KAR perception, in a MAX2-independent manner (Waters et al. 2015a), and this could be tested on eu-KAI2 clade proteins. As with the two other tested clades, (FK) and (HIL), none of the interaction assays revealed any preferential binding of GR24 isomers, despite the use of pure enantiomers. Indeed, only PpKAI2L-K showed similar binding affinity with both (-)-GR24 and (+)-GR24, but no stereoselectivity. Unfortunately, none of the PpKAI2L proteins from the (GJM) clade could be purified for interaction assays, which was also reported by (Bürger et al. 2019). In the future, 
overexpression in $P$. patens or in other heterologous systems (yeast, insect cells) may be a

567 solution for producing these proteins and carrying out biochemistry studies.

\section{PpKAI2L-H is the most efficient hydrolase among PpKAI2L proteins}

570 The PpKAI2L-H protein showed stronger cleavage activity towards both GR24 stereoisomers

571 as well as towards the synthetic probe $( \pm)$-GC242 compared to any other PpKAI2L protein, but 572 also compared to the Arabidopsis AtKAI2 and AtD14 proteins. Mutating the $\mathrm{Leu}^{28}$ residue into 573 a Phe was sufficient to reduce the efficiency of this activity (strong reduction of the $k_{\text {cat }}$ and of 574 the $V_{\max }$ ), but had no effect on the $K_{1 / 2}$ towards ( \pm )-GC242. The efficient cleavage activity of 575 PpKAI2L-H is therefore not likely due to a stronger affinity of this protein for the substrate. It 576 has been hypothesized that the $\mathrm{Leu}^{28}$ residue (like the Phe ${ }^{181}$ residue), which is unique to 577 PpKAI2L-H, does not particularly enlarge the pocket size of PpKAI2L-H (Bürger et al. 2019).

578 Therefore, our results may highlight the ability of a residue to control the exit of the cleavage reaction product. Indeed, the conserved $\mathrm{Phe}^{28}$ residue in D14/KAI2 proteins may act as a gate keeper, and this could explain the single turnover kinetics observed with some SL analogs (de Saint Germain et al. 2016). We hypothesize that the high velocity of PpKAI2L-H may be linked to the lack of this gate keeper residue, allowing a high substrate turnover. The stronger enzyme activity of PpKAI2L-H could have a specific role in $P$. patens, perhaps as a catabolic enzyme, to regulate the levels of bioactive signaling molecules (Seto et al. 2019).

585 When only the PpKAI2L- $H$ gene was mutated ( $\Delta h$ mutant), no striking phenotype was observed 586 (Figure 7), and in particular, the phenotypic response to (+)-GR24, which mimics PpCCD8587 derived SLs, was similar to that of WT plants (Figure 9D). In red light, however, the 588 gametophores of $\Delta h \boldsymbol{i}$ and $\Delta h \boldsymbol{i l}$ mutants were less elongated than WT gametophores, which was 589 also observed in Ppccd8 (Figure 8). However, in Ppccd8, this could be related to the higher 590 number of filaments, leading to the initiation of more (but smaller) gametophores, whereas the 591 number of filaments in the dark was not higher in mutants from the (HIL) clade (Figure 9 and 592 Table 1). In addition, these mutants even tended to have fewer filaments than WT (Figure 9 D593 E and Supplemental Figure S13A). Thus, mutants from the (HIL) clade are quite different from $594 P p c c d 8$, and the corresponding genes PpKAI2L-H $-I$ and $-L$ likely do not encode receptors for 595 PpCCD8-derived compounds. Notably, our results do not support a likely role for PpKAI2L-H 596 in the KL pathway, even though the binding of $\mathrm{KAR}_{1}$ by this protein was reported (Bürger et 597 al. 2019). Indeed, the $\Delta h$ and higher-order mutants ( $\Delta h i$ and $\Delta h i l$ ) tended to be smaller than WT 
in white light (Figure 7D and Table 1), but also showed a slight reduction in gametophore height in red light (Figure 8A), in contrast to Ppmax2-1 and eu-KAI2 clade mutants (Table 1). These findings suggest that (HIL) clade genes play a specific role in P. patens development. The association of this role with PpKAI2L-H enzyme activity remains to be discovered.

602

The Arabidopsis Atd14-1 kai2-2 mutant complementation assay provides important clues about PpKAI2L activity

Using the endogenous AtD14 promoter, we confirmed that expression of the PpKAI2L-C protein does not complement the Arabidopsis D14 function in rosette branching, as previously reported using the $35 \mathrm{~S}$ promoter (Bürger et al. 2019). We can now extend this observation to PpKAI2L-D, PpKAI2L-F, PpKAI2L-G, and PpKAI2L-J. Using the endogenous AtKAI2 promoter, we also confirmed that PpKAI2L-C cannot complement the kai2-2 mutation, as observed by (Bürger et al. 2019). Furthermore, we extended this observation to PpKAI2L-D and PpKAI2L-J. However, expressing moss PpKAI2L-G reduced the hypocotyl size and led to

612 larger cotyledons than in the double mutant Atd14-1 kai2-2 (Supplemental Figure S9C), 613 suggesting that PpKAI2L-G may be able to perceive and transduce the endogenous KL signal, 614 even though it does not respond to (-)-GR24. Similarly, when expressed in Arabidopsis, one 615 of the two KAI2 homologs from S. moellendorffi (SmKAI2b) partially complemented the kai2 616 mutant, whereas the other homolog and both the KAI2 homologs from M. polymorpha did not 617 restore the kai2 mutant phenotypes (Waters et al. 2015b). Strikingly, when expressed under the 618 control of the AtKAI2 promoter in the Arabidopsis Atd14-1 kai2-2 mutant, the moss PpKAI2L619 C or -D proteins exacerbated the defect induced by the kai2-2 mutation by leading to even more 620 elongated hypocotyls (for each construct, in one line out of two, Supplemental Figure S9B). 621 This suggests a putative interaction of these proteins with the Arabidopsis KAI2/KL pathway, which should be further investigated. Still, it is clear that none of the PpKAI2L proteins fully complements the AtD14 or KAI2 function, likely due to defective interactions with AtMAX2 and/or other components of SL/KL pathways.

Genetic analysis suggests that genes from the eu-KAI2 clade are involved in the PpMAX2-dependent pathway Mutant phenotypes clearly distinguish the eu-KAI2 clade from the three other clades (Table 1). Indeed, the quintuple eu-KAI2 mutant shows a phenotype in white light quite similar to that of 
630 Ppmax2-1. It also has elongated gametophores under red light, and few caulonema filaments in 631 the dark, suggesting that eu-KAI2 and PpMAX2 proteins could be members of the same 632 pathway (Figure 11). As eu-KAI2 proteins preferentially bind to the (-)-GR24 enantiomer, we 633 expected the mutants in this clade to be blind to (-)-GR24 application. This is what we observed 634 when transcriptional response markers were examined, with transcript levels remaining 635 unchanged following (-)-GR24 application in both the Ppmax2-1 and the quintuple eu-KAI2 636 clade mutant, but increasing in Ppccd8 (but not in WT, Figure 10 and Table 1). However, as 637 mentioned above, (-)-GR24 does not appear to be a perfect mimic of the unknown moss KL, 638 and other transcriptional response markers need to be found that would better reflect the moss $639 \mathrm{KL}$ response. As for the phenotypic response, the application of $0.1 \mu \mathrm{M}(-)$-GR2 4 had no effect on caulonema number in the dark in the eu-KAI2 clade mutants or in the WT, but it significantly decreased caulonema number in both Ppccd8 and Ppmax2-1 (Figure 9A). Strikingly, a $1 \mu \mathrm{M}$ concentration of (-)-GR24, which had the opposite effect on WT (increased caulonema number) and Ppccd8 (decreased caulonema number), produced no response in the Ppmax2-1 mutant, while the quintuple eu-KAI2 mutant showed a significant decrease in caulonema number under this treatment (Supplemental Figure S13). Thus, the quintuple eu-KAI2 mutant is still able to perceive (-)-GR24, as is the Ppmax2-1 mutant. As mentioned above, we hypothesize that other PpKAI2L proteins (presumably members of the (GJM) clade, see below) may perceive (-)-GR24, triggering the PpCCD8-derived compounds pathway (Figure 11). This does not rule out the possibility that eu-KAI2 clade and PpMAX2 proteins function in the same pathway. In addition, (-)-GR24 was reported to have a dual effect, promoting both the KAI2 and the D14 pathways in Arabidopsis roots (Villaecija-Aguilar et al. 2019).

652

Finally, both phenotypic responses in the dark and transcriptional responses to the (+)-GR24 enantiomer were unaffected in the eu-KAI2 clade mutants (Table 1), indicating that eu-KAI2 proteins are not receptors for PpCCD8-derived compounds.

PpKAI2L- G, -J, and -M mediate PpCCD8-derived (SL-related) responses

658 In white light (Figure 7), mutants affecting (FK) and (GJM) clades showed phenotypes 659 intermediate between those of WT and Ppccd8. In the dark under control conditions (Figure 9), 660 the caulonema numbers of mutants in the (FK) and (GJM) clades were also intermediate 661 between those of WT and Ppccd8 (see also Table 1). Based on the hypothesis that synthesis 662 and response mutants show similar phenotypes, genes from the (FK) and (GJM) clades are thus 
663 the best candidates for PpCCD8-derived compound receptors. When the phenotypic responses 664 of these mutants to (+)-GR24 application were examined, plants with KO mutations in PpKAI2L-J or PpKAI2L-G/M no longer responded to this compound (Figure 9C-D, $\boldsymbol{j}$, and $\boldsymbol{g} \boldsymbol{j} \boldsymbol{m}$ 1, and Supplemental Figure S13D, gjm-3, gjm-5, and gjm-4). By contrast, both $\Delta h f k j-1$ and $667 \Delta h f k j-2$ mutants showed a significant response to (+)-GR24 application (Figure 9D and 668 Supplemental Figure S13C), indicating that KO mutations in both PpKAI2L-F and PpKAI2L$669 K$, or the deletion of PpKAI2L-H, do not abolish the response to the PpCCD8-derived compound mimic, not even additively. The absence of a response in higher-order mutants where either PpKAI2L-J or PpKAI2L-G were knocked out confirms the prominent roles of both genes in the response to (+)-GR24. However, while as expected, the transcript levels of the Pp3c6_15020 response marker gene did not change in either $\boldsymbol{j}$ and $\boldsymbol{g} j \mathrm{~m}-2$ mutants following (+)GR24 application (Figure 10), the transcript levels of the PpKUF1LA gene increased in the $\boldsymbol{j}$ mutant, suggesting a response to the SL analog. Thus, while the KO mutation of either PpKAI2L-J or PpKAI2L-G was sufficient to abolish the phenotypic response in the dark, it did not completely abolish the transcriptional response to (+)-GR24. Presumably, the mutation of both $P p K A I 2 L-J$ and $-G$ or even all three genes $(-G-J-M)$ is necessary to fully impair this response. Another possibility is that the transcriptional markers, first identified using $( \pm)$-GR24 (Lopez-Obando et al. 2016a; Lopez-Obando et al. 2018) and Supplemental Figure S14), are not fully specific for assaying the responses to enantiomers. This result could also suggest that the transcriptional response, which was assessed far earlier than the phenotypic response (6 hours versus 15 days), is perhaps more sensitive to a very slight activation of the PpCCD8-derived SL pathway by PpKAI2L proteins.

PpKAI2L proteins are likely receptors in two separate pathways, one dependent and the other independent of the F-box protein PpMAX2

Our previous study of the F-box protein PpMAX2 indicated that, in contrast to its homolog in angiosperms, it is not involved in the response to PpCCD8-derived compounds (Lopez-Obando et al. 2018). Like MAX2 in angiosperms, however, PpMAX2 plays a role in early gametophore development and photomorphogenesis. We suggested that PpMAX2 could play a role in the moss KL signalling pathway, but we lacked evidence for other actors in this pathway in $P$. patens. The present study suggests that eu-KAI2 clade proteins are $\alpha / \beta$ hydrolases involved in the same pathway as PpMAX2, since mutating these genes resulted in similar light-related 
696 preventing us from obtaining further evidence that eu-KAI2 clade proteins are receptors of the

697 moss KL (Figure 11). Still, these results are consistent with the view that the KL pathway is

698 ancestral relative to the SL pathway, and that the ancestral role of MAX2 in the land plants 699 lineage is the transduction of the KL signal (Bythell-Douglas et al. 2017; Walker et al. 2019).

700 Such a KL pathway has been recently reported in M. polymorpha, further supporting this view $701 \quad$ (Mizuno et al. 2021).

702

PpKAI2L-J and PpKAI2L-G proteins are likely receptors of PpCCD8-derived compounds, 704 which we suspect to be non-canonical SLs. Strikingly, these receptors are not particularly more 705 similar to D14 than other PpKAI2L proteins. As hypothesized earlier (Lopez-Obando et al. 706 2016a; Bythell-Douglas et al. 2017), the expansion of the PpKAI2L family in P. patens (and 707 not in other bryophytes such as M. polymorpha, which contains two MpKAI2 genes), as in 708 parasitic angiosperms (Conn et al. 2015; Toh et al. 2015), may have allowed the emergence of 709 SL sensitivity (de Saint Germain et al. 2021b). Neofunctionalization of additional KAI2 copies 710 in $P$. patens ancestry towards SL perception is therefore a possible explanation for our findings 711 in this moss and would reveal a parallel evolutionary process, relative to the emergence of D14

712 in seed plants. We can also imagine that these neofunctionalized PpKAI2L proteins lost the 713 ability to interact with MAX2 in P. patens, and established a different protein network that 714 potentially integrates new factors such as an alternative F-box protein (Figure 11). The 715 remaining question is therefore to determine how SL signal transduction is achieved 716 downstream of perception by (GJM) clade PpKAI2L proteins.

717

718 Consequently, the search for proteins that interact with $P$. patens KL and PpCCD8-derived 719 compound receptors should be a priority in the near future. In particular, since SMXL proteins 720 are key members of both the SL and KL pathways in angiosperms and of the KL pathway in 721 M. polymorpha (Soundappan et al. 2015; Wang et al. 2015; Khosla et al. 2020; Mizuno et al. 722 2021), the specific involvement of PpSMXL homologs (four genes) is currently under 723 investigation by our group.

724

725 METHODS

726 Plant materials and growth conditions 
727 The Physcomitrium (Physcomitrella) patens Gransden wild-type (WT) strain was grown as

728 previously described (Hoffmann et al. 2014; Lopez-Obando et al. 2018) under long day (16h)

729 conditions. Unless otherwise stated in the legends, the experiments were always carried out on

$730 \mathrm{PpNO}_{3}$ medium (minimal medium described by Ashton et al., 1979) under the following control

731 conditions: $25^{\circ} \mathrm{C}$ during the day and $23{ }^{\circ} \mathrm{C}$ at night, $50 \%$ humidity, quantum irradiance of $\sim 80$

$732 \mu \mathrm{mol} / \mathrm{m}^{2} / \mathrm{s}$ using OSRAM L 36W/865 LUMILUX Cool daylight fluorescent tubes. Tissues

733 from young protonema fragments were multiplied prior to every experiment under the same

734 conditions but using medium with higher nitrogen content $\left(\mathrm{PpNH}_{4}\right.$ medium, $\mathrm{PpNO}_{3}$ medium

735 supplemented with $2.7 \mathrm{mM} \mathrm{NH}_{4}$ tartrate). For red light experiments, plants were grown on

$736 \mathrm{PpNO}_{3}$ medium in Magenta pots at $25{ }^{\circ} \mathrm{C}$, in continuous red light $\left(\sim 45 \mu \mathrm{mol} \mu \mathrm{mol} / \mathrm{m}^{2} / \mathrm{s}\right)$.

\section{Germination assays of Phelipanche ramosa seeds}

738 P. patens WT plants were grown on $\mathrm{PpNH}_{4}$ plates with cellophane disks for two weeks. The

739 plants (and cellophane) were transferred onto low-phosphate $\mathrm{PpNO}_{3}$ medium (Phosphate buffer

740 was replaced with $1 \mathrm{~g} / \mathrm{L}$ of MES buffer and the $\mathrm{pH}$ adjusted to 5.8) for another two weeks. $P$.

741 patens exudates were collected by transferring the plants (still on cellophane disks) onto plates

742 containing $10 \mathrm{~mL}$ distilled water and incubating them in the growth chamber with gentle

743 agitation. After $48 \mathrm{~h}$, the exudates were pipeted and filtered $(0.2 \mu \mathrm{m})$. The exudates (Figure 1A)

744 were diluted twice prior to testing their germination-stimulating activity on preconditioned

745 seeds of parasitic P. ramosa plants, as described previously (Pouvreau et al. 2013; Pouvreau et

746 al. 2021). Distilled water was used as a negative control and $0.1 \mu \mathrm{M}( \pm)$-GR24 as a positive

747 control. For each $P$. ramosa group, germination stimulant activities were normalized with the

748 respective negative and positive controls $\left(\left(\mathrm{GS}_{-}-\mathrm{GS}^{-}\right) /\left(\mathrm{GS}^{+}-\mathrm{GS}^{-}\right)\right.$; with $\mathrm{GSs}^{-}$Germination

749 Stimulant activity of a bioassay, $\mathrm{GS}^{-}$: mean of GS activities of negative controls and $\mathrm{GS}^{+}$: mean

750 of GS activities of positive controls). For germination assays on plates (Figure 1B-C), WT and

751 Ppccd8 were cultivated as above, and preconditioned P. ramosa seeds were placed onto the

752 plates after 10 days of phosphate starvation. Germinated and non-germinated seeds were

753 counted on three plates, with 7-10 microscope fields per plate, five days after adding $P$. ramosa

754 seeds. Results are expressed as percentage of germination.

\section{CRISPR-Cas9 mediated mutagenesis and homologous recombination in $P$. patens}


756 P. patens mutants were obtained as described in (Lopez-Obando et al. 2016b), using CRISPR-

757 Cas9 technology. PpKAI2L coding sequences were used to search for CRISPR RNA (crRNA) 758 contiguous to a PAM motif recognized by Streptococcus pyogenes Cas9 (NGG), using the webtool CRISPOR V4 against the P. patens genome Phytozome V9 (http://crispor.tefor.net/).

760 Guide crRNAs were chosen in each PpKAI2L gene, preferably in the first exon, to ideally obtain 761 the earliest nonsense mutation possible. When no guide could be designed in the first exon, it 762 was alternatively chosen to recognize a region in close proximity to the codon for one of the 763 last two residues of the catalytic triad (Figure 3). The same crRNA was used to target $P p K A I 2 L-$ $764 G$ and $-M$. Small constructs containing each crRNA fused to either the proU6 or the proU3 765 snRNA promoters (Collonnier et al. 2017) between the attB1/attB2 Gateway recombination 766 sequences were synthesized by Twist Biosciences. These inserts were then cloned into 767 pDONR207 vectors (Invitrogen). Polyethylene glycol-mediated protoplast transformation was 768 performed with multiple pDONR207-sgRNA as described previously (Lopez-Obando et al. 769 2016b). Mutations in the PpKAI2L genes were confirmed by PCR amplification of PpKAI2L 770 loci around the recognition sequence of each guide RNA and by sequencing the PCR products.

771 The PpKAI2L-H $(\Delta h)$ deletion mutant was obtained through homologous recombination. The

772 full coding sequence of PpKAI2L-H from the ATG to stop was replaced with a resistance 773 cassette. A 550 bp PpKAI2L-H 5' CDS flanking sequence was cloned into the pBNRF vector 774 (Thelander et al. 2007) cut with BstBI/Xhol. Then a 500 bp PpKAI2L-H 3' CDS flanking 775 sequence was cloned into the BNRF-PpKAI2L-H 5' construct digested with BcuI, so that the 776 kanamycin resistance cassette of the vector was flanked by the PpKAI2L-H 5' and 3' 777 sequences. P. patens WT protoplasts were transformed with the resulting construct as described 778 previously (Lopez-Obando et al. 2016b) and transformants selected on $50 \mathrm{mg} / \mathrm{L}$ 779 Geneticin/G418. Transient expression of the CRE recombinase (Trouiller et al. 2006) in a 780 confirmed transformant removed the resistance cassette to obtain the Ppkai2L- $\Delta h$ mutant, as 781 described in Supplemental Figures S10 and S11.

\section{Phenotypic assays of $\boldsymbol{P}$. patens}

783 To measure diameters in the light, plants were grown in Petri dishes (6 to 8 plates per genotype), 784 starting from very small pieces of protonema, with 5 plants per plate, on $\mathrm{PpNO}_{3}$ medium 785 (Ashton et al. 1979) covered with cellophane disks as previously described (Hoffmann et al. 786 2014). Thus, between 30 and 40 plants were grown per genotype. After 5 weeks of growth, 
787 pictures of the plates were taken, and the ImageJ (http://imagej.nih.gov/ij/) manual selection

788 tool was used for each plant, allowing the area and Feret diameter to be measured. Analysis of 789 caulonema growth in the dark was performed in 24-well plates, starting from very small pieces 790 of protonema. For each genotype/treatment combination, 24 plants were grown, dispatched in

7913 different plates. These plants were grown for $\sim$ two weeks under control conditions before

792 being placed vertically ( \pm treatment) in the dark for $\sim 10$ days. A single picture of each well was 793 taken under Axio Zoom microscope (Zeiss) with a dedicated program. Caulonema were 794 counted and measured using ImageJ (http://imagej.nih.gov/ij/) (see also (Guillory and 795 Bonhomme 2021a)).

\section{Chemicals}

797 Racemic and pure enantiomers of GR24 and the ( \pm )-GC242 probe were produced by F-D Boyer 798 (ICSN, France) (de Saint Germain et al. 2021a). Racemic CL was kindly provided by A. 799 Scaffidi (University of Western Australia, Perth, Australia). Chemicals were diluted in DMSO 800 or acetone as indicated in the figure legends. $\mathrm{KAR}_{2}$ was purchased from Chiralix.

\section{$801 \quad$ RT-qPCR analysis}

802 Freshly ground WT (Gransden) tissues were inoculated in Petri dishes containing $\mathrm{PpNO}_{3}$ 803 medium, overlaid with a cellophane sheet. Protonema tissues were harvested after six days, 10 804 days or 15 days of growth under long-day conditions (see above). To obtain gametophores and 805 spores, plants regenerated from spores were cultivated for two weeks, transferred to Magenta 806 pots containing $\mathrm{PpNO}_{3}$ medium (nine plants per pot), and cultivated under the same conditions 807 as above. Gametophores were harvested after 35 days. Both protonema and gametophore 808 samples were flash frozen in liquid nitrogen and stored at $-80{ }^{\circ} \mathrm{C}$ until RNA extraction. At 35 809 days, the remaining pots were transferred to short-day conditions $\left(15^{\circ} \mathrm{C}, 100 \%\right.$ hygrometry, 810 quantum irradiance of $15 \mu \mathrm{mol} \mathrm{m}^{-2} \mathrm{~s}^{-1}$ ) for approximately two months until capsule maturity. 811 Capsules were surface sterilized (5 minutes in a mixture of $90 \%$ pure ethanol and 10\% sodium 812 hypochlorite solution) and rinsed with sterile water. Each of the four biological replicates 813 consisted of 10-20 capsules from which spores were freed by mechanical disruption and 814 separated from capsule debris by filtering through a $25 \mu \mathrm{m}$ nylon mesh. Spores were kept in 815 sterile water, flash frozen in liquid nitrogen, and stored at $-80^{\circ} \mathrm{C}$ until RNA extraction. For all 816 samples except spores, tissues were ground in liquid nitrogen using a mortar and pestle and 
817 RNA was extracted from the samples and treated with DNAse I using a Plant RNeasy Mini 818 extraction kit (Qiagen) following the manufacturer's instructions. Spores were recovered in 1 819 mL TRIzol reagent (Invitrogen) and crushed manually using fine pestles. RNA was separated 820 from cell debris and protein using chloroform, precipitated with isopropanol, and washed with

$82170 \%$ ethanol. The spore RNA pellets were dissolved in RLT buffer from a Qiagen Plant RNeasy 822 Mini kit and treated with DNAse I on columns following the manufacturer's instructions. 500 823 ng of each RNA sample was used for reverse-transcription using RevertAid H Minus Reverse 824 Transcriptase from ThermoFisher. The quality of the obtained cDNA extracts was verified by 825 RT-PCR using the reference gene $P p A P T$. Quantitative RT-PCR was carried out in a total 826 volume of $5 \mu \mathrm{L}$ using SsoAdvanced Universal SYBR Green Supermix from BioRad and the 827 following program on QuantStudio ${ }^{\mathrm{TM}} 5$ (ThermoFisher Scientific): initial denaturation at $95^{\circ} \mathrm{C}$ 828 for 3 minutes, then 45 cycles of $95{ }^{\circ} \mathrm{C}$ for 10 seconds and $60^{\circ} \mathrm{C}$ for 30 seconds. Using the CTi 829 (for the genes of interest) and CTref (mean for the two reference genes) values obtained, relative 830 expression (RE) was calculated as $R E=2-$ CTi/2-CTref.

\section{Constructs and generation of transgenic Arabidopsis lines}

832 The expression vectors for transgenic Arabidopsis were constructed using a MultiSite Gateway 833 Three-Fragment Vector Construction kit (Invitrogen). All of the PpKAI2L constructs were 834 tagged with a 6xHA epitope tag at their $\mathrm{C}$-terminus. The transgenic lines were resistant to 835 hygromycin. The AtD14 native promoter $(0.8 \mathrm{~kb})$ and $A t K A I 2$ native promoter $(0.7 \mathrm{~kb})$ were 836 amplified by PCR from Col-0 genomic DNA and cloned into $p D O N R-P 4 P 1 R$, using Gateway 837 recombination (Invitrogen) (see Supplemental Table S2 for primers). The AtD14 CDS and 838 AtKAI2 CDS were PCR amplified from Col-0 cDNA, and the PpKAI2L CDS were PCR 839 amplified from $P$. patens cDNA and recombined into pDONR221 (Invitrogen). 6xHA with a 840 linker (gift from U. Pedmale, Cold Spring Harbor Laboratory) was cloned into $p D O N R-P 2 R P 3$ 841 (Invitrogen). The suitable combination of promoters, CDS, and $6 x H A$ were cloned into the $842 \mathrm{pH} 7 \mathrm{~m} 34 \mathrm{GW}$ final destination vectors using the three fragment recombination system (Karimi 843 et al. 2007) and named pD14:CDS-6xHA or pKAI2:CDS-6xHA. The Arabidopsis d14-1 kai28442 double mutant in the Landsberg background (gift from M. Waters, University of Western 845 Australia Perth) was transformed following the conventional floral dip method (Clough and 846 Bent 1998), with Agrobacterium strain GV3101. For all constructs, more than 12 independent 847 T1 lines were isolated, and between 2-4 representative single-insertion lines were selected in 848 the T2 generation. Two lines per construct were shown in these analyses. The phenotypic 
849 analysis shown in Figure 6 and Supplemental Figure S9 was performed on segregating T3

850 homozygous lines.

851 Protein extraction and immunoblotting

852

853 Total protein extract was prepared from 8 to 10 ten-day-old Arabidopsis seedlings in Laemmli

854 buffer and boiled for $5 \mathrm{~min}$. Total protein were separated by 10\% SDS-PAGE and transferred 855 onto polyvinylidene difluoride membrane (Bio-Rad) probed with anti-HA primary antibody 856 (1:10000; SIGMA H9658-100UL Lot\#128M4789V) and then anti-mouse-IgG-HRP secondary

857 antibody (1:10000; SIGMA A9044-2ML-100UL Lot\#029M4799V). As a loading control, a 858 Ponceau staining or a Tubulin detection with anti-Tubulin primary antibody $(1: 10,000$; SIGMA 859 T5168-2ML Lot\#04M4760V) and then anti-mouse-IgG-HRP secondary antibody (1:10000; 860 SIGMA A9044-2ML-100UL Lot\#029M4799V) was used.

861

862 Arabidopsis hypocotyl elongation and cotyledon expansion assays

863 Arabidopsis seeds were surface sterilized by consecutive treatments of $5 \mathrm{~min}$ in $70 \%(\mathrm{v} / \mathrm{v})$ 864 ethanol and $0.05 \%(\mathrm{w} / \mathrm{v})$ sodium dodecyl sulfate (SDS), and $5 \mathrm{~min}$ in $95 \%$ (v/v) ethanol. Then 865 seeds were sown on $0.25 \mathrm{X}$ Murashige and Skoog (MS) medium (Duchefa Biochemie) 866 containing $1 \%$ agar, supplemented with $1 \mu \mathrm{M}(+)-G R 24,(-)-G R 24$ or with $0.01 \%$ DMSO 867 (control). For the hypocotyl elongation assay, seeds were stratified at $4{ }^{\circ} \mathrm{C}$ ( 2 days in the dark), 868 exposed to white light ( $\left.125-180 \mu \mathrm{mol} / \mathrm{m}^{2} / \mathrm{s}\right)$ for $3 \mathrm{~h}$, transferred to the dark for $21 \mathrm{~h}$, and exposed 869 to low light $\left(25-30 \mu \mathrm{mol} / \mathrm{m}^{2} / \mathrm{s}\right)$ for 10 days at $21{ }^{\circ} \mathrm{C}$. For the cotyledon expansion assay, 870 seedlings were grown in white light $\left(125-180 \mu \mathrm{mol} / \mathrm{m}^{2} / \mathrm{s}\right)$ for 4 days. Cotyledon area and 871 hypocotyl lengths were quantified using ImageJ (http://imagej.nih.gov/ij/)

\section{Quantification of Arabidopsis branching}

873 Experiments were carried out in the summer in a greenhouse under long photoperiods (15-16 $874 \mathrm{~h}$ per day); daily temperatures fluctuated between $18{ }^{\circ} \mathrm{C}$ and $25^{\circ} \mathrm{C}$. Peak PAR levels were 875 between 700 and $1000 \mu \mathrm{mol} \mathrm{m} \mathrm{m}^{-2} \mathrm{~s}^{-1}$. Plants were watered twice a week with tap water. The 876 number of rosette branches longer than $5 \mathrm{~mm}$ was counted when the plants were 40 days old.

\section{Protein expression and purification}


878 AtD14, RMS3, and AtKAI2 with cleavable GST tags were expressed and purified as described 879 in (de Saint Germain et al. 2016). For PpKAI2L protein expression, the full-length CDS from 880 P. patens were amplified by PCR using cDNA template and specific primers (see Supplemental 881 Table S2) containing a protease cleavage site for tag removal, and subsequently cloned into the 882 pGEXT-4T-3 expression vector. For PpKAI2L-L, the N-terminal 47 amino acids were 883 removed. The expression and purification of PpKAI2L proteins followed the same method as 884 for AtD14 and AtKAI2.

\section{Site-directed mutagenesis}

886 Site-directed mutagenesis of $P p K A I 2 L-H$ was performed using a QuickChange II XL Site 887 Directed Mutagenesis kit (Stratagene), performed on pGEX-4T-3-PpKAI2L-H (see 888 Supplemental Table S2 for primers). Mutagenesis was verified by DNA sequencing.

\section{Enzymatic degradation of GR24 isomers by purified proteins}

890 The ligand $(10 \mu \mathrm{M})$ was incubated with and without purified RMS3/AtKAI2/PpKAI2L proteins $891(5 \mu \mathrm{M})$ for $150 \mathrm{~min}$ at $25^{\circ} \mathrm{C}$ in PBS $(0.1 \mathrm{~mL}, \mathrm{pH}=6.8)$ in the presence of $( \pm)$-1-indanol $(100$ $892 \mu \mathrm{M})$ as an internal standard. The solutions were acidified to $\mathrm{pH}=1$ by adding trifluoroacetic 893 acid $(2 \mu \mathrm{L})$ to quench the reaction and centrifuged (12 $\mathrm{min}, 12,000 \mathrm{rpm})$. The samples were 894 then subjected to RP-UPLC-MS analyses. The instrument used for all the analyses was an Ultra 895 Performance Liquid Chromatography system equipped with a PDA and Triple Quadrupole 896 mass spectrometer Detector (Acquity UPLC-TQD, Waters, USA). RP-UPLC (HSS C 18 column, $8971.8 \mu \mathrm{m}, 2.1 \mathrm{~mm} \times 50 \mathrm{~mm}$ ) was carried out with $0.1 \%$ formic acid in $\mathrm{CH}_{3} \mathrm{CN}$ and $0.1 \%$ formic 898 acid in water (aq. FA, $0.1 \%$, v/v, pH 2.8 ) as eluents $\left[10 \% \mathrm{CH}_{3} \mathrm{CN}\right.$, followed by a linear gradient 899 from 10 to $100 \%$ of $\left.\mathrm{CH}_{3} \mathrm{CN}(4 \mathrm{~min})\right]$ at a flow rate of $0.6 \mathrm{~mL} / \mathrm{min}$. The detection was performed 900 by PDA and using the TQD mass spectrometer operated in Electrospray ionization positive 901 mode at $3.2 \mathrm{kV}$ capillary voltage. The cone voltage and collision energy were optimized to

902 maximize the signal, with a cone voltage of $20 \mathrm{~V}$ and collision energy of $12 \mathrm{eV}$. The collision 903 gas was argon at a pressure maintained near $4.5 .10^{-3} \mathrm{mBar}$.

\section{$904 \quad$ Enzymatic kinetic assays}


905 Enzyme assays with pro-fluorescent probes and $p$-nitrophenyl acetate were performed as

906 described in (de Saint Germain et al. 2016), using a TriStar LB 941 Multimode Microplate

907 Reader from Berthold Technologies.

908 nanoDifferential Scanning Fluorimetry (nanoDSF)

909 Proteins were diluted in Phosphate buffer saline (PBS) (100 mM Phosphate, pH 6.8, $150 \mathrm{mM}$

$910 \mathrm{NaCl})$ to $\sim 10 \mu \mathrm{M}$ concentration. Ligand was tested at the concentration of $200 \mu \mathrm{M}$. The

911 intrinsic fluorescence signal was measured as a function of increasing temperature in a

912 Prometheus NT.48 fluorimeter (Nanotemper ${ }^{\mathrm{TM}}$ ), with 55\% excitation light intensity and 1

$913{ }^{\circ} \mathrm{C} /$ minute temperature ramp. Analyses were performed on capillaries filled with $10 \mu \mathrm{L}$ of the

914 respective samples. The intrinsic fluorescence signal was expressed as the $350 \mathrm{~nm} / 330 \mathrm{~nm}$

915 emission ratio, which increases as the proteins unfold, and was plotted as a function of

916 temperature. The plots show one of the three independent data collections that were performed

917 for each protein.

918 Intrinsic tryptophan fluorescence assays

919 Intrinsic tryptophan fluorescence assays and determination of the dissociation constant $K_{\mathrm{d}}$ were 920 performed as described in (de Saint Germain et al. 2016), using a Spark ${ }^{\circledR}$ Multimode 921 Microplate Reader from Tecan.

922 Direct electrospray ionization - mass spectrometry of PpKAI2L proteins (ESI)-MS under 923 denaturing conditions

924 Mass spectrometry measurements were performed with an electrospray Q-TOF mass 925 spectrometer (Waters) equipped with a Nanomate device (Advion, Inc.). The HD_A_384 chip 926 (5 $\mu \mathrm{m}$ I.D. nozzle chip, flow rate range $100-500 \mathrm{~nL} / \mathrm{min}$ ) was calibrated before use. For 927 ESI-MS measurements, the Q-TOF instrument was operated in RF quadrupole mode with the 928 TOF data being collected between $\mathrm{m} / \mathrm{z} 400-2990$. Collision energy was set to $10 \mathrm{eV}$, and argon 929 was used as the collision gas. PpKAI2L proteins $(50 \mu \mathrm{M})$ in $50 \mathrm{mM}$ ammonium acetate $(\mathrm{pH}$ 930 6.8) in the presence or absence of GR24 enantiomers $(500 \mu \mathrm{M})$ were incubated for $10 \mathrm{~min}$ at 931 room temperature before denaturation in 50\% acetonitrile and $1 \%$ formic acid. The solutions 932 were directly injected for Mass spectra acquisition or digested before LC-MS/MS analyses. 
933 Mass Lynx version 4.1 (Waters) and Peakview version 2.2 (Sciex) software were used for data

934 acquisition and processing, respectively. Deconvolution of multiply charged ions was

935 performed by applying the MaxEnt algorithm (Sciex). The average protein masses were 936 annotated in the spectra, and the estimated mass accuracy was \pm 2 Da. External calibration was

937 performed with $\mathrm{NaI}$ clusters $\left(2 \mu \mathrm{g} / \mu \mathrm{L}\right.$, isopropanol/ $\mathrm{H}_{2} \mathrm{O} 50 / 50$, Waters $)$ in the acquisition $\mathrm{m} / \mathrm{z}$

938 mass range.

\section{Homology modeling}

940 Figure 5B showing a superimposition model was prepared using PyMOL (DeLano Scientific)

941 with the crystal structures of AtD14 (PDB ID 4IH4 ) and PpKAI2L-H (PDB ID 6AZD).

\section{Statistical analyses}

943 ANOVA, Kruskal-Wallis, Mann-Whitney, post-hoc Dunnett, post-hoc Dunn, and Tukey

944 multiple comparisons tests (details in figure legends) were carried out either in $\mathrm{R}$ version 3.6.3

945 or in GraphPad Prism version 8.4.2. Unless otherwise defined, the statistical significance scores

946 used were as follow: ${ }^{*} 0.01 \leq \mathrm{P}<0.05, * * 0.001 \leq \mathrm{P}<0.01, * * * \mathrm{P}<0.001$. Same letters scores indicate

947 that $\mathrm{P} \geq 0.05$ (non-significant differences). All statistical analyses are described in Supplemental

$948 \quad$ File S1.

Accession numbers

950 Sequence data from this article can be found in the GenBank/Phytozome libraries under the 951 accession numbers described in Supplemental Table S3.

952 Table 1 Overview of Ppkai2L mutant phenotypes

953

\begin{tabular}{|c|c|c|c|c|c|c|c|}
\hline \multirow[t]{2}{*}{ Genotypes } & \multirow[t]{2}{*}{ Diameter } & \multirow{2}{*}{$\begin{array}{c}\text { Gametophore } \\
\text { size }\end{array}$} & \multirow{2}{*}{$\begin{array}{c}\text { Caulonema } \\
\text { number }\end{array}$} & \multicolumn{2}{|c|}{ Response to (+)-GR24 } & \multicolumn{2}{|c|}{ Response to (-)-GR24 } \\
\hline & & & & $\begin{array}{c}\text { Caulonema } \\
\text { number }\end{array}$ & $\begin{array}{l}\text { Transcriptional } \\
\text { markers }\end{array}$ & $\begin{array}{l}\text { Caulonema } \\
\text { number }\end{array}$ & $\begin{array}{c}\text { Transcriptional } \\
\text { markers }\end{array}$ \\
\hline & (Figure 7) & $\begin{array}{c}\text { (Figure 8, } \\
\text { Supplemental } \\
\text { Figure S12) }\end{array}$ & $\begin{array}{l}\text { (Figure 2, 9, } \\
\text { Supplemental } \\
\text { Figure S13) }\end{array}$ & $\begin{array}{l}\text { (Figure 2, 9, } \\
\text { Supplemental } \\
\text { Figure S13) }\end{array}$ & (Figure 10) & $\begin{array}{l}\text { (Figure 2, 9, } \\
\text { Supplemental } \\
\text { Figure S13) }\end{array}$ & (Figure 10) \\
\hline WT & WT & WT & WT & $y$ & $\pi$ & $\pi$ or $=$ & $=$ \\
\hline Ppccd8 & $>$ WT & $<W T$ & $>\mathrm{WT}$ & ע & $\pi$ & $=$ or $y$ & $\pi$ \\
\hline Ppmax2-1 & $<W T$ & $>$ WT & $<W T$ & $y$ & $=$ & $=$ or $y$ & $=$ \\
\hline eu-kai2 & $<W T$ & $>$ WT & $\leq W T$ & ע & $\pi$ & $=$ or $y$ & $=$ \\
\hline$(f k)^{*}$ & $\geq \mathrm{WT}$ & $\approx W T$ & $\geq W T$ & ע & Not tested & $=$ & Not tested \\
\hline
\end{tabular}




\begin{tabular}{|c|c|c|c|c|c|c|c|}
\hline (hil) & $\leq W T$ & $\leq \mathrm{WT}$ & $\leq \mathrm{WT}$ & ע & Not tested & $=$ & Not tested \\
\hline (gjm) & $\geq W T$ & $\leq \mathrm{WT}$ & $>\mathrm{WT}$ & $=$ & $\pi$ or $=$ & $=$ or $y$ & or $=$ \\
\hline
\end{tabular}

\section{Supplemental Data}

Supplemental Figure S1. Sequence alignment of Physcomitrium patens (Pp) PpKAI2L proteins with AtD14 and AtKAI2 proteins from Arabidopsis thaliana (At).

960 Supplemental Figure S2. Expression of $P p K A I 2 L$ genes throughout $P$. patens vegetative 961 development.

962 Supplemental Figure S3. Nano Differential Scanning Fluorimetry (nanoDSF) analysis of the 963 PpKAI2L protein response to GR24 isomers.

964 Supplemental Figure S4. Intrinsic tryptophan fluorescence shows that GR24 isomers bind to 965 PpKAI2L proteins with different affinities.

966 Supplemental Figure S5. PpKAI2L hydrolysis activity towards $p$-NPA.

967 Supplemental Figure S6. PpKAI2L enzymatic activities confirm stereoselectivity.

968 Supplemental Figure S7. Characterization of ( \pm )-GC242 profluorescent probe activity on 969 moss and PpKAI2L enzymatic activities towards ( \pm )-GC242.

970 Supplemental Figure S8. Mass spectrometry characterization of covalent PpKAI2L-ligand 971 complexes.

972 Supplemental Figure S9. Complementation assays of the Arabidopsis Atd14-1 kai2-2 double 973 mutant.

974 Supplemental Figure S10. Mutations obtained in all 13 PpKAI2L genes.

975 Supplemental Figure S11. The Ppkai2L- $\Delta$ h mutant. 
976 Supplemental Figure S12. Gametophore heights of Ppkai2L mutants in red light.

977 Supplemental Figure S13. Phenotypic response of Ppkai2L mutants to (-)-GR24 and (+)-

978 GR24 application: caulonema number in the dark.

979 Supplemental Figure S14. Transcriptional response of WT and Ppccd8 to ( \pm )-GR24

980 application: use of the Pp3c6_15020 marker gene.

981 Supplemental Table S1. Mutants used in this study.

982 Supplemental Table S2. Oligonucleotides used in this study.

983 Supplemental Table S3. List of gene sequences used in this study.

984 Supplemental File S1. Results of statistical tests.

\section{ACKNOWLEDGEMENTS}

986 The authors thank Adrian Scaffidi (University of Western Australia, Perth, Australia) for the 987 gift of carlactone, and Mark Waters (University of Western Australia, Perth, Australia) for 988 Arabidopsis kai2-2 and d14-1 kai2-2 mutants. We are grateful to Jean-Paul Pillot (IJPB) for 989 precious help with Arabidopsis branching assays, to Pauline Rode for help with genotyping, 990 and to Fabien Nogué (IJPB) for stimulating discussions. MLO thanks Eva Sundberg for her 991 support on his SL/KL's activities at SLU.

992 This research was supported by the Agence Nationale de la Recherche (contract ANR-12993 BSV6-004-01). The IJPB benefits from the support of the Labex Saclay Plant Sciences-SPS 994 (ANR-10-LABX-0040-SPS). This work was supported by a "Infrastructures en Biologie Santé 995 et Agronomie" grant to SICAPS platform of the Institute for Integrative Biology of the Cell, 996 and CHARM3AT Labex program (ANR-11-LABX-39) for ICSN. A.d.S.G. is the recipient of 997 an AgreenSkills award from the European Union in the framework of the Marie-Curie FP7

998 COFUND People Programme and fellowship from Saclay Plant Sciences (ANR-17-EUR999 0007). This work has benefited from the facilities and expertise of the I2BC proteomic platform 
1000 (Proteomic-Gif, SICaPS) supported by IBiSA, Ile de France Region, Plan Cancer, CNRS and

1001 Paris-Sud University.

1002

1003

\section{AUTHOR CONTRIBUTIONS}

1004

1005

1006

1007

1008

1009

1010

1011

1012

1013

1014

1015

1016

1017

1018

1019

1020

1021

1022

1023

1024

1025

1026

1027

1028

1029

1030

1031

1032

1033

1034

1035

1036

1037

1038

1039

1040

1041

SB, AdSG, ML-O and CR designed the project. ML-O, AG, F-DB, DC, BH, PLB, J-BP, AdSG and SB conducted experiments. AG, ML-O, F-DB, DC, PLB, J-BP, PD, CR, AdSG and $\mathrm{SB}$ analyzed the data. AG and SB wrote the manuscript, with essential contributions from ML-O, F-DB, PD, CR and AdSG.

\section{REFERENCES}

Akiyama K, Matsuzaki K, Hayashi H (2005) Plant sesquiterpenes induce hyphal branching in arbuscular mycorrhizal fungi. Nature 435 (7043):824-827. doi:10.1038/nature03608

Akiyama K, Ogasawara S, Ito S, Hayashi H (2010) Structural requirements of strigolactones for hyphal branching in AM fungi. Plant Cell Physiol 51 (7):1104-1117. doi: $10.1093 / \mathrm{pcp} / \mathrm{pcq} 058$

Al-Babili S, Bouwmeester HJ (2015) Strigolactones, a novel carotenoid-derived plant hormone. Annu Rev Plant Biol 66:161-186. doi:10.1146/annurev-arplant-043014-114759

Alder A, Jamil M, Marzorati M, Bruno M, Vermathen M, Bigler P, Ghisla S, Bouwmeester H, Beyer P, Al-Babili S (2012) The path from beta-carotene to carlactone, a strigolactonelike plant hormone. Science 335 (6074):1348-1351. doi:10.1126/science.1218094

Arite T, Umehara M, Ishikawa S, Hanada A, Maekawa M, Yamaguchi S, Kyozuka J (2009) d14, a strigolactone-insensitive mutant of rice, shows an accelerated outgrowth of tillers. Plant Cell Physiol 50 (8):1416-1424. doi:10.1093/pcp/pcp091

Ashton NW, Grimsley NH, Cove DJ (1979) Analysis of gametophytic development in the moss, Physcomitrella patens , using auxin and cytokinin resistant mutants. Planta 144 (5):427-435. doi:10.1007/bf00380118

Besserer A, Puech-Pages V, Kiefer P, Gomez-Roldan V, Jauneau A, Roy S, Portais JC, Roux C, Becard G, Sejalon-Delmas N (2006) Strigolactones stimulate arbuscular mycorrhizal fungi by activating mitochondria. PLoS Biol 4 (7):e226

Blázquez MA, Nelson DC, Weijers D (2020) Evolution of Plant Hormone Response Pathways. Annu Rev Plant Biol 71:327-353. doi:10.1146/annurev-arplant-050718-100309

Boutet-Mercey S, Perreau F, Roux A, Clave G, Pillot JP, Schmitz-Afonso I, Touboul D, Mouille G, Rameau C, Boyer FD (2018) Validated Method for Strigolactone Quantification by Ultra High-Performance Liquid Chromatography - Electrospray Ionisation Tandem Mass Spectrometry Using Novel Deuterium Labelled Standards. Phytochem Anal 29 (1):59-68. doi:10.1002/pca.2714

Bowman JL, Briginshaw LN, Florent SN (2019) Evolution and co-option of developmental regulatory networks in early land plants. Curr Top Dev Biol 131:35-53. doi:10.1016/bs.ctdb.2018.10.001

Boyer FD, de Saint Germain A, Pillot JP, Pouvreau JB, Chen VX, Ramos S, Stevenin A, Simier P, Delavault P, Beau JM, Rameau C (2012) Structure-activity relationship studies of 
1042

1043

1044

1045

1046

1047

1048

1049

1050

1051

1052

1053

1054

1055

1056

1057

1058

1059

1060

1061

1062

1063

1064

1065

1066

1067

1068

1069

1070

1071

1072

1073

1074

1075

1076

1077

1078

1079

1080

1081

1082

1083

1084

1085

1086

1087

1088

1089

1090

strigolactone-related molecules for branching inhibition in garden pea: molecule design for shoot branching. Plant Physiol 159 (4):1524-1544. doi:10.1104/pp.112.195826

Bürger M, Chory J (2020) The Many Models of Strigolactone Signaling. Trends Plant Sci 25 (4):395-405. doi:10.1016/j.tplants.2019.12.009

Bürger M, Mashiguchi K, Lee HJ, Nakano M, Takemoto K, Seto Y, Yamaguchi S, Chory J (2019) Structural Basis of Karrikin and Non-natural Strigolactone Perception in Physcomitrella patens. Cell Rep 26 (4):855-865.e855. doi:10.1016/j.celrep.2019.01.003

Bythell-Douglas R, Rothfels CJ, Stevenson DWD, Graham SW, Wong GK, Nelson DC, Bennett T (2017) Evolution of strigolactone receptors by gradual neo-functionalization of KAI2 paralogues. BMC biology 15 (1):52. doi:10.1186/s12915-017-0397-z

Clough SJ, Bent AF (1998) Floral dip: a simplified method for Agrobacterium-mediated transformation of Arabidopsis thaliana. Plant J 16 (6):735-743. doi:10.1046/j.1365313x.1998.00343.x

Collonnier C, Guyon-Debast A, Maclot F, Mara K, Charlot F, Nogué F (2017) Towards mastering CRISPR-induced gene knock-in in plants: Survey of key features and focus on the model Physcomitrella patens. Methods 121-122:103-117. doi:10.1016/j.ymeth.2017.04.024

Conn CE, Bythell-Douglas R, Neumann D, Yoshida S, Whittington B, Westwood JH, Shirasu K, Bond CS, Dyer KA, Nelson DC (2015) PLANT EVOLUTION. Convergent evolution of strigolactone perception enabled host detection in parasitic plants. Science 349 (6247):540-543. doi:10.1126/science.aab1140

Conn CE, Nelson DC (2015) Evidence that KARRIKIN-INSENSITIVE2 (KAI2) Receptors may Perceive an Unknown Signal that is not Karrikin or Strigolactone. Front Plant Sci 6:1219. doi:10.3389/fpls.2015.01219

Cook CE, Whichard LP, Turner B, Wall ME, Egley GH (1966) Germination of Witchweed (Striga lutea Lour.): Isolation and Properties of a Potent Stimulant. Science 154 (3753):1189-1190

Coudert Y, Palubicki W, Ljung K, Novak O, Leyser O, Harrison CJ (2015) Three ancient hormonal cues co-ordinate shoot branching in a moss. Elife 4. doi:10.7554/eLife.06808

de Saint Germain A, Clave G, Badet-Denisot MA, Pillot JP, Cornu D, Le Caer JP, Burger M, Pelissier F, Retailleau P, Turnbull C, Bonhomme S, Chory J, Rameau C, Boyer FD (2016) An histidine covalent receptor and butenolide complex mediates strigolactone perception. Nat Chem Biol 12 (10):787-794. doi:10.1038/nchembio.2147

de Saint Germain A, Clavé G, Boyer FD (2021a) Synthesis of Profluorescent Strigolactone Probes for Biochemical Studies. In: Prandi C, Cardinale F (eds) Methods Mol Biol, vol 2309. 2021/05/25 edn. Springer US, pp 219-231. doi:10.1007/978-1-0716-1429-7_17

de Saint Germain A, Jacobs A, Brun G, Pouvreau J-B, Braem L, Cornu D, Clavé G, Baud̄u E, Steinmetz V, Servajean V, Wicke S, Gevaert K, Simier P, Goormachtig S, Delavault P, Boyer F-D (2021b) A Phelipanche ramosa KAI2 Protein Perceives enzymatically Strigolactones and Isothiocyanates. Plant Comm. doi:10.1016/j.xplc.2021.100166

de Saint Germain A, Retailleau P, Norsikian S, Servajean V, Pelissier F, Steinmetz V, Pillot JP, Rochange S, Pouvreau JB, Boyer FD (2019) Contalactone, a contaminant formed during chemical synthesis of the strigolactone reference GR24 is also a strigolactone mimic. Phytochemistry 168:112112. doi:10.1016/j.phytochem.2019.112112

Decker EL, Alder A, Hunn S, Ferguson J, Lehtonen MT, Scheler B, Kerres KL, Wiedemann G, Safavi-Rizi V, Nordzieke S, Balakrishna A, Baz L, Avalos J, Valkonen JPT, Reski $\mathrm{R}$ (2017) Strigolactone biosynthesis is evolutionarily conserved, regulated by phosphate starvation and contributes to resistance against phytopathogenic fungi in a moss, Physcomitrella patens. New Phytol 216 (2):455-468. doi:10.1111/nph.14506 
1091

1092

1093

1094

1095

1096

1097

1098

1099

1100

1101

1102

1103

1104

1105

1106

1107

1108

1109

1110

1111

1112

1113

1114

1115

1116

1117

1118

1119

1120

1121

1122

1123

1124

1125

1126

1127

1128

1129

1130

1131

1132

1133

1134

1135

1136

1137

1138

1139

Delaux PM, Xie X, Timme RE, Puech-Pages V, Dunand C, Lecompte E, Delwiche CF, Yoneyama K, Becard G, Sejalon-Delmas N (2012) Origin of strigolactones in the green lineage. New Phytol 195 (4):857-871. doi:10.1111/j.1469-8137.2012.04209.x

Delavault P, Montiel G, Brun G, Pouvreau JB, Thoiron S, Simier P (2017) Communication Between Host Plants and Parasitic Plants. Adv Bot Res 82:55-82. doi:0.1016/bs.abr.2016.10.006

Floková K, Shimels M, Andreo Jimenez B, Bardaro N, Strnad M, Novák O, Bouwmeester HJ (2020) An improved strategy to analyse strigolactones in complex sample matrices using UHPLC-MS/MS. Plant Methods 16:125. doi:10.1186/s13007-020-00669-3

Gomez-Roldan V, Fermas S, Brewer PB, Puech-Pages V, Dun EA, Pillot JP, Letisse F, Matusova R, Danoun S, Portais JC, Bouwmeester H, Becard G, Beveridge CA, Rameau C, Rochange SF (2008) Strigolactone inhibition of shoot branching. Nature 455 (7210):189-194. doi:10.1038/nature07271

Guillory A, Bonhomme S (2021a) Methods for Medium-Scale Study of Biological Effects of Strigolactone-Like Molecules on the Moss Physcomitrium (Physcomitrella) patens. In: Prandi C, Cardinale F (eds) Methods Mol Biol, vol 2309. 2021/05/25 edn. Springer US, pp 143-155. doi:10.1007/978-1-0716-1429-7_12

Guillory A, Bonhomme S (2021b) Phytohormone biosynthesis and signaling pathways of mosses. Plant Mol Biol. doi:10.1007/s11103-021-01172-6

Gutjahr C, Gobbato E, Choi J, Riemann M, Johnston MG, Summers W, Carbonnel S, Mansfield C, Yang SY, Nadal M, Acosta I, Takano M, Jiao WB, Schneeberger K, Kelly KA, Paszkowski U (2015) Rice perception of symbiotic arbuscular mycorrhizal fungi requires the karrikin receptor complex. Science 350 (6267):1521-1524. doi:10.1126/science.aac9715

Hamiaux C, Drummond RS, Janssen BJ, Ledger SE, Cooney JM, Newcomb RD, Snowden KC (2012) DAD2 is an alpha/beta hydrolase likely to be involved in the perception of the plant branching hormone, strigolactone. Curr Biol 22 (21):2032-2036. doi:10.1016/j.cub.2012.08.007

Harris BJ, Harrison CJ, Hetherington AM, Williams TA (2020) Phylogenomic Evidence for the Monophyly of Bryophytes and the Reductive Evolution of Stomata. Curr Biol 30 (11):2001-2012.e2002. doi:10.1016/j.cub.2020.03.048

Hoffmann B, Proust H, Belcram K, Labrune C, Boyer FD, Rameau C, Bonhomme S (2014) Strigolactones inhibit caulonema elongation and cell division in the moss Physcomitrella patens. PLoS One 9 (6):e99206. doi:10.1371/journal.pone.0099206

Huet S, Pouvreau J-B, Delage E, Delgrange S, Marais C, Bahut M, Delavault P, Simier P, Poulin L (2020) Populations of the Parasitic Plant Phelipanche ramosa Influence Their Seed Microbiota. Front Plant Sci 11 (1075). doi:10.3389/fpls.2020.01075

Jamil M, Kountche BA, Wang JY, Haider I, Jia KP, Takahashi I, Ota T, Asami T, Al-Babili S (2020) A New Series of Carlactonoic Acid Based Strigolactone Analogs for Fundamental and Applied Research. Front Plant Sci 11:434. doi:10.3389/fpls.2020.00434

Karimi M, Bleys A, Vanderhaeghen R, Hilson P (2007) Building blocks for plant gene assembly. Plant Physiol 145 (4):1183-1191. doi:10.1104/pp.107.110411

Khosla A, Morffy N, Li Q, Faure L, Chang SH, Yao J, Zheng J, Cai ML, Stanga JP, Flematti GR, Waters M, Nelson DC (2020) Structure-Function Analysis of SMAX1 Reveals Domains that Mediate its Karrikin-Induced Proteolysis and Interaction with the Receptor KAI2. Plant Cell. doi:10.1105/tpc.19.00752

Li W, Nguyen KH, Chu HD, Watanabe Y, Osakabe Y, Sato M, Toyooka K, Seo M, Tian L, Tian C, Yamaguchi S, Tanaka M, Seki M, Tran LP (2020) Comparative functional 
analyses of DWARF14 and KARRIKIN INSENSITIVE 2 in drought adaptation of Arabidopsis thaliana. Plant J. doi:10.1111/tpj.14712

Lopez-Obando M, Conn CE, Hoffmann B, Bythell-Douglas R, Nelson DC, Rameau C, Bonhomme S (2016a) Structural modelling and transcriptional responses highlight a clade of PpKAI2-LIKE genes as candidate receptors for strigolactones in Physcomitrella patens. Planta 243 (6):1441-1453. doi:10.1007/s00425-016-2481-y

Lopez-Obando M, de Villiers R, Hoffmann B, Ma L, de Saint Germain A, Kossmann J, Coudert Y, Harrison CJ, Rameau C, Hills P, Bonhomme S (2018) Physcomitrella patens MAX2 characterization suggests an ancient role for this F-box protein in photomorphogenesis $\begin{array}{lllll}\text { rather than strigolactone signalling. New Phytol } 219 & \text { (2):743-756. }\end{array}$ doi:10.1111/nph.15214

Lopez-Obando M, Hoffmann B, Géry C, Guyon-Debast A, Téoulé E, Rameau C, Bonhomme S, Nogué F (2016b) Simple and Efficient Targeting of Multiple Genes Through CRISPR-Cas9 in Physcomitrella patens. G3 (Bethesda) 6 (11):3647-3653. doi: $10.1534 / \mathrm{g} 3.116 .033266$

Lopez-Obando M, Ligerot Y, Bonhomme S, Boyer F-D, Rameau C (2015) Strigolactone biosynthesis and signaling in plant development. Development 142 (21):3615-3619. doi:10.1242/dev.120006

Mizuno Y, Komatsu A, Shimazaki S, Naramoto S, Inoue K, Xie X, Ishizaki K, Kohchi T, Kyozuka J (2021) Major components of the KARRIKIN INSENSITIVE2-dependent signaling pathway are conserved in the liverwort Marchantia polymorpha. Plant Cell. doi:10.1093/plcell/koab106

Nelson DC, Scaffidi A, Dun EA, Waters MT, Flematti GR, Dixon KW, Beveridge CA, Ghisalberti EL, Smith SM (2011) F-box protein MAX2 has dual roles in karrikin and strigolactone signaling in Arabidopsis thaliana. Proc Natl Acad Sci U S A 108 (21):8897-8902. doi:10.1073/pnas.1100987108

Ortiz-Ramirez C, Hernandez-Coronado M, Thamm A, Catarino B, Wang M, Dolan L, Feijo JA, Becker JD (2016) A Transcriptome Atlas of Physcomitrella patens Provides Insights into the Evolution and Development of Land Plants. Mol Plant 9 (2):205-220. doi:10.1016/j.molp.2015.12.002

Perroud PF, Haas FB, Hiss M, Ullrich KK, Alboresi A, Amirebrahimi M, Barry K, Bassi R, Bonhomme S, Chen H, Coates JC, Fujita T, Guyon-Debast A, Lang D, Lin J, Lipzen A, Nogué F, Oliver MJ, Ponce de León I, Quatrano RS, Rameau C, Reiss B, Reski R, Ricca M, Saidi Y, Sun N, Szövényi P, Sreedasyam A, Grimwood J, Stacey G, Schmutz J, Rensing SA (2018) The Physcomitrella patens gene atlas project: large-scale RNA-seq based expression data. Plant J 95 (1):168-182. doi:10.1111/tpj.13940

Pouvreau JB, Gaudin Z, Auger B, Lechat MM, Gauthier M, Delavault P, Simier P (2013) A high-throughput seed germination assay for root parasitic plants. Plant Methods 9 (1):32. doi:10.1186/1746-4811-9-32

Pouvreau JB, Poulin L, Huet S, Delavault P (2021) Strigolactone-Like Bioactivity via Parasitic Plant Germination Bioassay. In: Prandi C, Cardinale F (eds) Methods Mol Biol, vol 2309. 2021/05/25 edn. Springer US, pp 59-73. doi:10.1007/978-1-0716-1429-7_6

Proust H, Hoffmann B, Xie X, Yoneyama K, Schaefer DG, Nogue F, Rameau C (2011) Strigolactones regulate protonema branching and act as a quorum sensing-like signal in the moss Physcomitrella patens. Development 138 (8):1531-1539. doi: $10.1242 /$ dev.058495

Puttick MN, Morris JL, Williams TA, Cox CJ, Edwards D, Kenrick P, Pressel S, Wellman CH, Schneider H, Pisani D, Donoghue PCJ (2018) The Interrelationships of Land Plants and the Nature of the Ancestral Embryophyte. Curr Biol 28 (5):733-745.e732. doi:10.1016/j.cub.2018.01.063 
Rial C, Varela RM, Molinillo JMG, López-Ráez JA, Macías FA (2019) A new UHPLC-MS/MS method for the direct determination of strigolactones in root exudates and extracts. Phytochem Anal 30 (1):110-116. doi:10.1002/pca.2796

Scaffidi A, Waters MT, Sun YK, Skelton BW, Dixon KW, Ghisalberti EL, Flematti GR, Smith SM (2014) Strigolactone Hormones and Their Stereoisomers Signal through Two Related Receptor Proteins to Induce Different Physiological Responses in Arabidopsis. Plant Physiol 165 (3):1221-1232. doi:10.1104/pp.114.240036

Seto Y, Yasui R, Kameoka H, Tamiru M, Cao M, Terauchi R, Sakurada A, Hirano R, Kisugi T, Hanada A, Umehara M, Seo E, Akiyama K, Burke J, Takeda-Kamiya N, Li W, Hirano Y, Hakoshima T, Mashiguchi K, Noel JP, Kyozuka J, Yamaguchi S (2019) Strigolactone perception and deactivation by a hydrolase receptor DWARF14. Nat Commun 10 (1):191. doi:10.1038/s41467-018-08124-7

Shabek N, Ticchiarelli F, Mao H, Hinds TR, Leyser O, Zheng N (2018) Structural plasticity of D3-D14 ubiquitin ligase in strigolactone signalling. Nature 563 (7733):652-656. doi:10.1038/s41586-018-0743-5

Soundappan I, Bennett T, Morffy N, Liang Y, Stanga JP, Abbas A, Leyser O, Nelson D (2015) SMAX1-LIKE/D53 Family Members Enable Distinct MAX2-Dependent Responses to Strigolactones and Karrikins in Arabidopsis. Plant Cell 27 (11):3143-3159. doi:10.1105/tpc. 15.00562

Stojanova B, Delourme R, Duffé P, Delavault P, Simier P (2019) Genetic differentiation and host preference reveal non-exclusive host races in the generalist parasitic weed Phelipanche ramosa. Weed Research 59 (2):107-118. doi:10.1111/wre.12353

Thelander M, Nilsson A, Olsson T, Johansson M, Girod PA, Schaefer DG, Zryd JP, Ronne H (2007) The moss genes PpSKI1 and PpSKI2 encode nuclear SnRK1 interacting proteins with homologues in vascular plants. Plant Mol Biol 64 (5):559-573. doi:10.1007/s11103-007-9176-5

Toh S, Holbrook-Smith D, Stogios PJ, Onopriyenko O, Lumba S, Tsuchiya Y, Savchenko A, McCourt P (2015) Structure-function analysis identifies highly sensitive strigolactone receptors in Striga. Science 350 (6257):203-207. doi:10.1126/science.aac9476

Trouiller B, Schaefer DG, Charlot F, Nogue F (2006) MSH2 is essential for the preservation of genome integrity and prevents homeologous recombination in the moss Physcomitrella patens. Nucleic Acids Res 34 (1):232-242. doi:10.1093/nar/gkj423

Umehara M, Hanada A, Yoshida S, Akiyama K, Arite T, Takeda-Kamiya N, Magome H, Kamiya Y, Shirasu K, Yoneyama K, Kyozuka J, Yamaguchi S (2008) Inhibition of shoot branching by new terpenoid plant hormones. Nature 455 (7210):195-200. doi: 10.1038 /nature 07272

Villaecija-Aguilar JA, Hamon-Josse M, Carbonnel S, Kretschmar A, Schmidt C, Dawid C, Bennett T, Gutjahr C (2019) SMAX1/SMXL2 regulate root and root hair development downstream of KAI2-mediated signalling in Arabidopsis. PLoS genetics 15 (8):e1008327. doi:10.1371/journal.pgen.1008327

Walker CH, Siu-Ting K, Taylor A, O'Connell MJ, Bennett T (2019) Strigolactone synthesis is ancestral in land plants, but canonical strigolactone signalling is a flowering plant innovation. BMC biology 17 (1):70. doi:10.1186/s12915-019-0689-6

Wang L, Wang B, Jiang L, Liu X, Li X, Lu Z, Meng X, Wang Y, Smith SM, Li J (2015) Strigolactone Signaling in Arabidopsis Regulates Shoot Development by Targeting D53-Like SMXL Repressor Proteins for Ubiquitination and Degradation. Plant Cell 27 (11):3128-3142. doi:10.1105/tpc. 15.00605

Wang L, Waters MT, Smith SM (2018) Karrikin-KAI2 signalling provides Arabidopsis seeds with tolerance to abiotic stress and inhibits germination under conditions unfavourable to seedling establishment. New Phytol 219 (2):605-618. doi:10.1111/nph.15192 
Waters MT, Gutjahr C, Bennett T, Nelson DC (2017) Strigolactone Signaling and Evolution. Annu Rev Plant Biol 68:291-322. doi:10.1146/annurev-arplant-042916-040925

Waters MT, Nelson DC, Scaffidi A, Flematti GR, Sun YK, Dixon KW, Smith SM (2012) Specialisation within the DWARF14 protein family confers distinct responses to karrikins and strigolactones in Arabidopsis. Development 139 (7):1285-1295. doi:10.1242/dev.074567

Waters MT, Scaffidi A, Flematti G, Smith SM (2015a) Substrate-Induced Degradation of the alpha/beta-Fold Hydrolase KARRIKIN INSENSITIVE2 Requires a Functional Catalytic Triad but Is Independent of MAX2. Mol Plant 8 (5):814-817. doi:10.1016/j.molp.2014.12.020

Waters MT, Scaffidi A, Moulin SL, Sun YK, Flematti GR, Smith SM (2015b) A Selaginella moellendorffii Ortholog of KARRIKIN INSENSITIVE2 Functions in Arabidopsis Development but Cannot Mediate Responses to Karrikins or Strigolactones. Plant Cell 27 (7):1925-1944. doi:10.1105/tpc. 15.00146

Xie X (2016) Structural diversity of strigolactones and their distribution in the plant kingdom. J Pestic Sci 41 (4):175-180. doi:10.1584/jpestics.J16-02

Yao J, Scaffidi A, Meng Y, Melville KT, Komatsu A, Khosla A, Nelson DC, Kyozuka J, Flematti GR, Waters MT (2021) Desmethyl butenolides are optimal ligands for karrikin receptor proteins. New Phytol 230 (3):1003-1016. doi:10.1111/nph.17224

Yao R, Ming Z, Yan L, Li S, Wang F, Ma S, Yu C, Yang M, Chen L, Chen L, Li Y, Yan C, Miao D, Sun Z, Yan J, Sun Y, Wang L, Chu J, Fan S, He W, Deng H, Nan F, Li J, Rao Z, Lou Z, Xie D (2016) DWARF14 is a non-canonical hormone receptor for strigolactone. Nature 536 (7617):469-473. doi:10.1038/nature19073

Yoneyama K (2020) Recent progress in the chemistry and biochemistry of strigolactones. J Pestic Sci. doi:doi:10.1584/jpestics.D19-084

Yoneyama K, Mori N, Sato T, Yoda A, Xie X, Okamoto M, Iwanaga M, Ohnishi T, Nishiwaki H, Asami T, Yokota T, Akiyama K, Yoneyama K, Nomura T (2018a) Conversion of carlactone to carlactonoic acid is a conserved function of MAX1 homologs in strigolactone biosynthesis. New Phytol 218 (4):1522-1533. doi:10.1111/nph.15055

Yoneyama K, Xie X, Yoneyama K, Kisugi T, Nomura T, Nakatani Y, Akiyama K, McErlean CSP (2018b) Which are the major players, canonical or non-canonical strigolactones? J Exp Bot 69 (9):2231-2239. doi:10.1093/jxb/ery090

Zheng J, Hong K, Zeng L, Wang L, Kang S, Qu M, Dai J, Zou L, Zhu L, Tang Z, Meng X, Wang B, Hu J, Zeng D, Zhao Y, Cui P, Wang Q, Qian Q, Wang Y, Li J, Xiong G (2020) Karrikin Signaling Acts Parallel to and Additively with Strigolactone Signaling to Regulate Rice Mesocotyl Elongation in Darkness. Plant Cell 32 (9):2780-2805. doi:10.1105/tpc.20.00123 
A

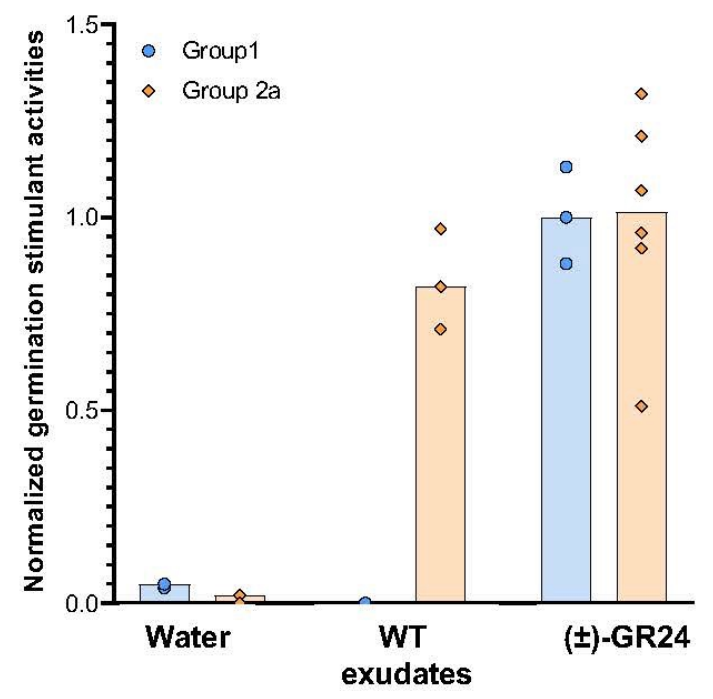

C
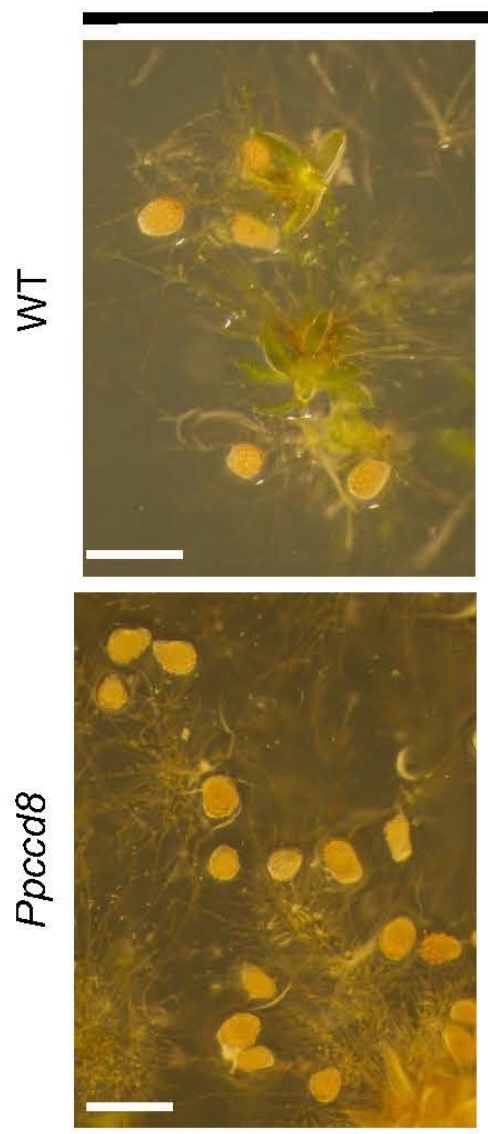

B

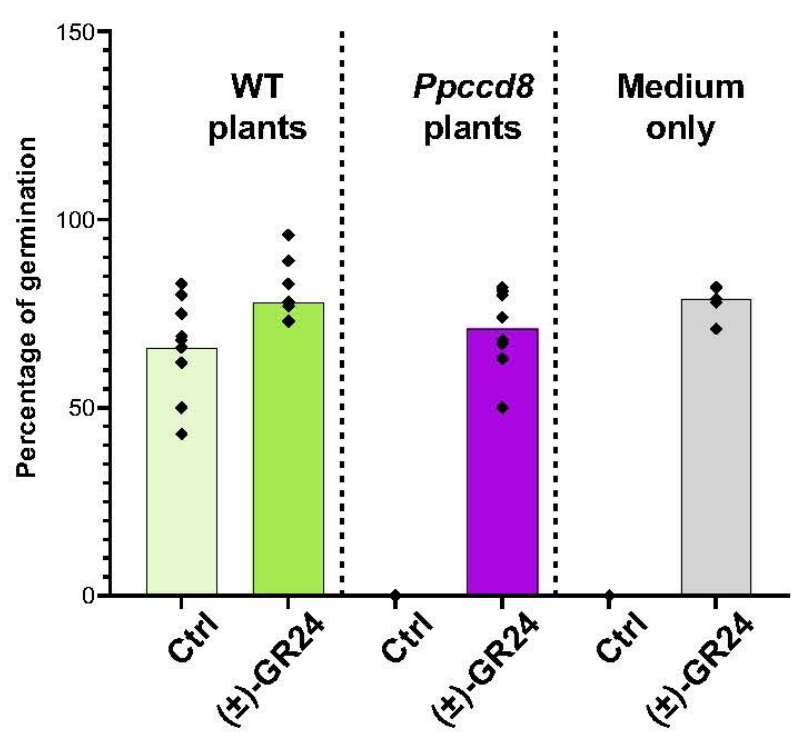

Group 2a
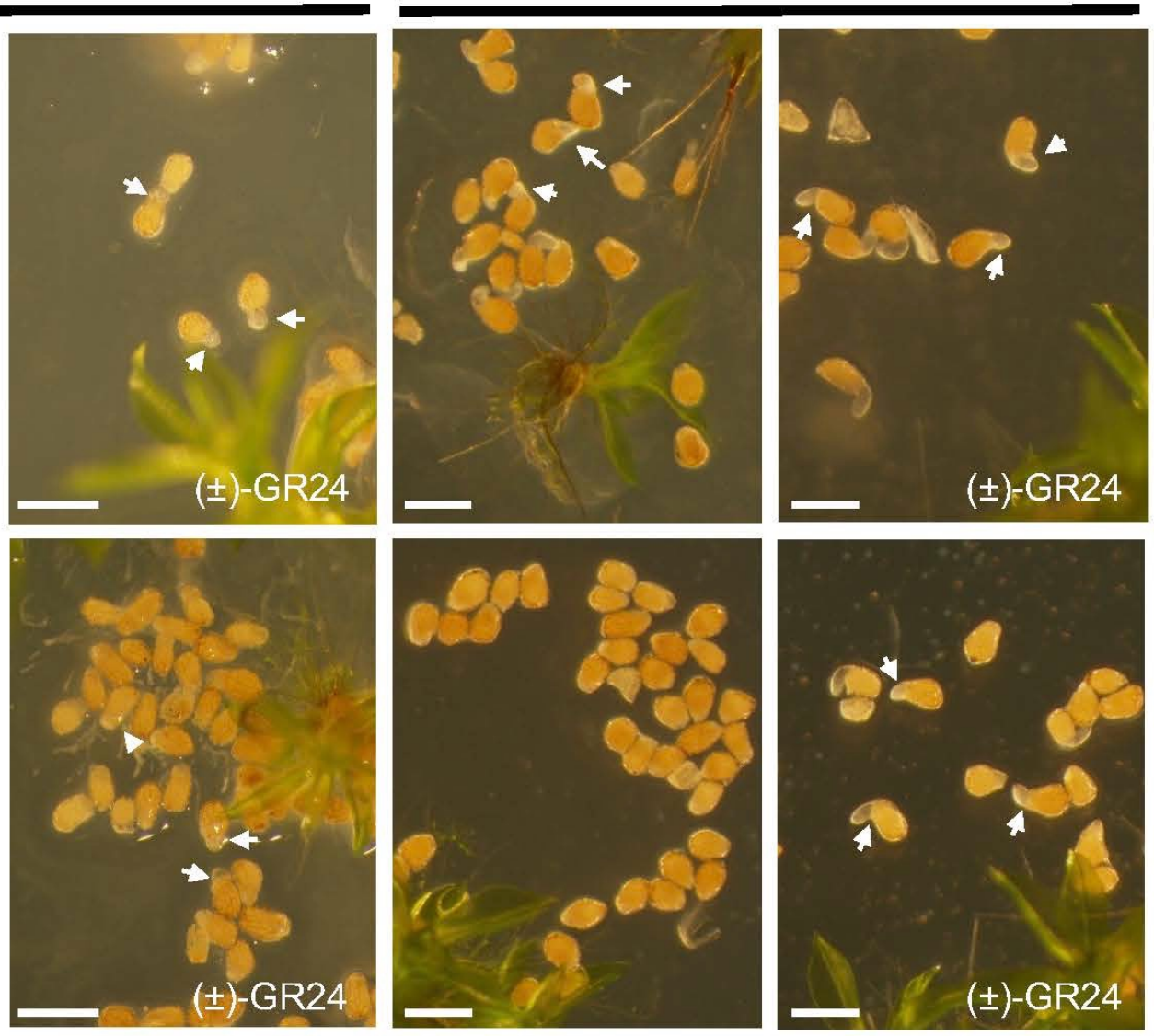

Figure 1. PpCCD8-derived compounds are germination stimulants (GS) of a specific group of Phelipanche ramosa.

(A) Germination Stimulant activities of $P$. patens exudates on $P$. ramosa group 1 and 2 a seeds relative to $0.1 \mu \mathrm{M}( \pm)$ GR24. $(n=6)$. (B) Percentage of germination of seeds of $P$. ramosa group $2 a$ on plates with $P$. patens WT, Ppccd8 mutant plants, or with culture medium only, with or without $0.1 \mu \mathrm{M}( \pm)$-GR24 $(\mathrm{n}=6)$. (C) Seeds from $P$. ramosa group 1 (left) and group 2a (right) on plates with WT (top) or Ppccd8 mutant plants (bottom), with or without $0.1 \mu \mathrm{M}( \pm)$-GR24. Arrows indicate germinating seeds. Scale bar $=0.5 \mathrm{~mm}$. 

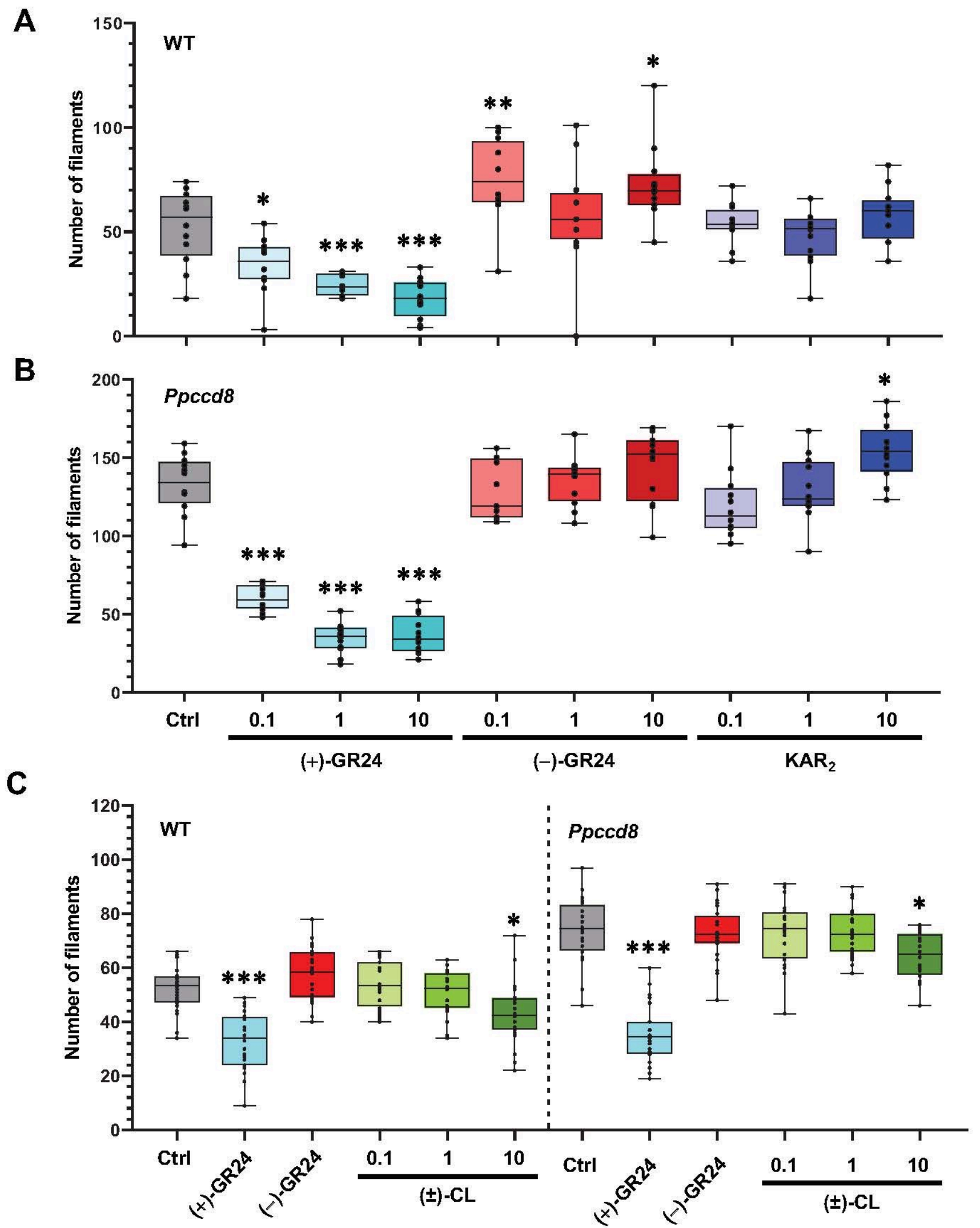

Figure 2. Phenotypic response to (+)- and (-)-GR24 enantiomers and natural compounds: number of caulonema filaments.

Caulonema filaments were counted for WT (A) and the Ppccd8 SL synthesis mutant (B) grown for 10 days vertically in the dark, following application of increasing concentrations $(0.1,1$ and $10 \mu \mathrm{M})$ of $(+)$-GR24 (blue boxes), (-)-GR24 (red boxes) and $\mathrm{KAR}_{2}$, (dark blue boxes). Control (Ctrl): $0.01 \%$ DMSO. (C) Caulonema filament numbers of WT and Ppccd8 mutant grown for 10 days vertically in the dark, with increasing concentrations $(0.1,1$ and $10 \mu \mathrm{M})$ of $( \pm)$-CL (green boxes). Control (Ctrl): $0.01 \%$ DMSO. (+)-GR24 (blue boxes) and (-)-GR24 (red boxes) were applied at $1 \mu \mathrm{M}$. Significant differences between control and treated plants within a genotype based on an ANOVA, followed by a Dunnett post-hoc test for multiple comparisons: ${ }^{* * *} \mathrm{P}<0.001$; ${ }^{* *} \mathrm{P}<0.01$; ${ }^{*} \mathrm{P}<0.05$; For each genotype/treatment combination, $\mathrm{n}=24$ plants grown in three different well-plates. Whiskers refer to minimum and maximum values, bars inside the boxplot to the median. 


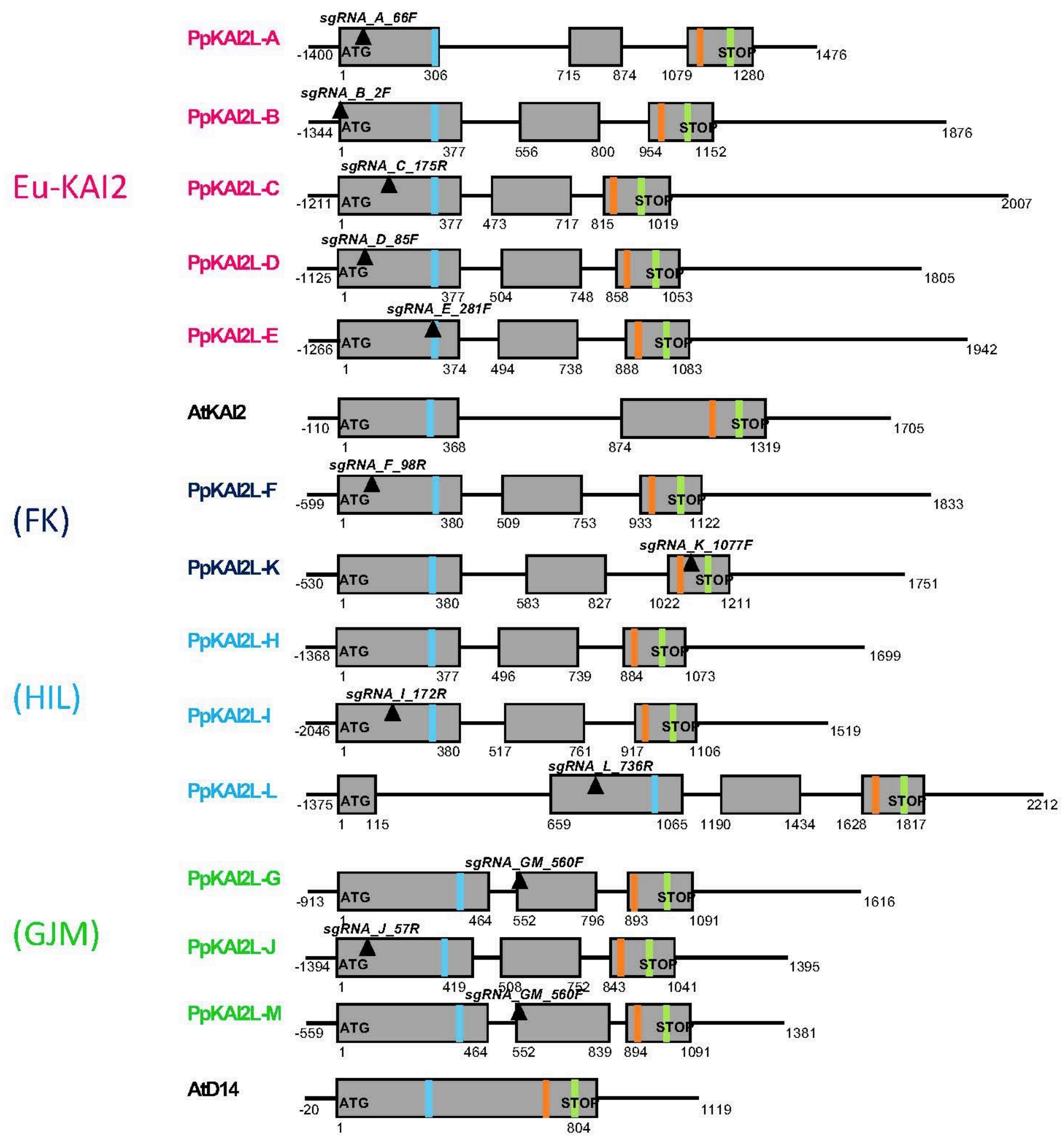

Figure 3. Gene models of the PpKAI2L gene family showing the catalytic triad and position of crRNAs.

Genes are presented as organized in subclades. Exons are displayed as grey boxes, introns and UTRs are depicted as thin black lines. Start and Stop codons are written in bold, while plain text indicates the start/end position for each feature, relative to the start codon. Only 5'-UTR regions are not represented true to scale. Transcript versions that were used are V3.1 (downloaded from the Phytozome website in September 2019) for all PpKAI2L genes except for PpKAI2L-B, PpKAI2L$H$ and PpKAI2L-M (V3.2). Regions targeted by crRNAs are indicated as black inverted triangles, with their names written in bold italic. Light blue, orange and light green bands represent respectively the codons for the $\mathrm{S}, \mathrm{D}$ and $\mathrm{H}$ residues of the catalytic triad (see Supplemental Table S3 for reference sequences). 

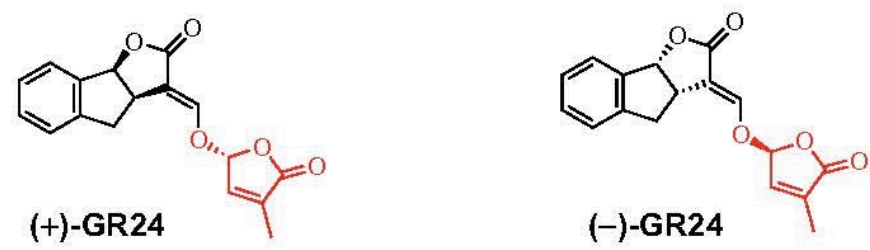

$( \pm)-G C 242$

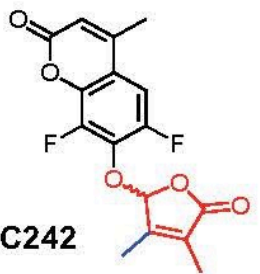

B

\begin{tabular}{|c|c|c|c|c|c|c|c|c|c|c|}
\hline \multirow{3}{*}{$\begin{array}{c}\text { Related figure } \\
\text { Ligand }\end{array}$} & \multirow{2}{*}{\multicolumn{3}{|c|}{ Prote in stability assay ${ }^{a}$}} & \multirow{2}{*}{\multicolumn{2}{|c|}{$\begin{array}{c}\text { Binding affinity determination } \\
(K d)^{b} \\
\text { Supplemental Figure S4 }\end{array}$}} & \multirow{2}{*}{\multicolumn{2}{|c|}{$\begin{array}{l}\text { GR24 cleavage assay } \\
\text { Supplemental Figure S7 }\end{array}$}} & \multirow{3}{*}{ 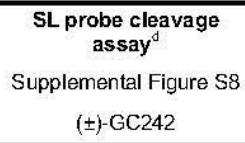 } & \multirow{2}{*}{\multicolumn{2}{|c|}{$\begin{array}{c}\begin{array}{c}\text { Covalent adduct } \\
\text { detection }\end{array} \\
\text { Supplemental Figure } \$ 9\end{array}$}} \\
\hline & & & & & & & & & & \\
\hline & Mock & (+)-GR24 & (-)-GR24 & (+)-GR24 & (-)-GR24 & $(+)-G R 24$ & (-)-GR24 & & $(+)-G R 24$ & (-)-GR24 \\
\hline AtKAI2 & $46.7^{\circ} \mathrm{C}$ & $46.6^{\circ} \mathrm{C}$ & $42.4^{\circ} \mathrm{C}$ & $\begin{array}{l}\text { No binding } \\
\text { detected }\end{array}$ & $45 \pm 9 \mu \mathrm{M}$ & $1.1 \%$ & $28.0 \%$ & Residual activity & No & Yes \\
\hline PpKAIZL-C & $49.7^{\circ} \mathrm{C}$ & $50.5^{\circ} \mathrm{C}$ & $42.9^{\circ} \mathrm{C}$ & Not tested & Not tested & $5.3 \%$ & $21.3 \%$ & Residual activity & No & Yes \\
\hline PpKAI2L-D & $49.9^{\circ} \mathrm{C}$ & $49.5^{\circ} \mathrm{C}$ & $39.9^{\circ} \mathrm{C}$ & $\begin{array}{l}\text { No binding } \\
\text { detected }\end{array}$ & $92 \pm 10 \mu \mathrm{M}$ & $0.3 \%$ & $16.2 \%$ & Residual activity & No & Yes \\
\hline PpKAI2L-E & $53.2^{\circ} \mathrm{C}$ & $54.0^{\circ} \mathrm{C}$ & $42.3^{\circ} \mathrm{C}$ & $\begin{array}{l}\text { No binding } \\
\text { detected }\end{array}$ & $39 \pm 10 \mu \mathrm{M}$ & $1.3 \%$ & $20.5 \%$ & Residual activity & No & Yes \\
\hline PpKAI2L-F & $44.0^{\circ} \mathrm{C}$ & $44.6^{\circ} \mathrm{C}$ & $45.0^{\circ} \mathrm{C}$ & $\begin{array}{l}\text { No binding } \\
\text { detected }\end{array}$ & No binding detected & $4.1 \%$ & $4.9 \%$ & Residual activity & No & $\begin{array}{l}\text { Small } \\
\text { amount }\end{array}$ \\
\hline PpKAIZL-K & $44.9^{\circ} \mathrm{C}$ & No signal & No signal & $107 \pm 11 \mu \mathrm{M}$ & $41 \pm 43 \mu \mathrm{M}$ & $2.2 \%$ & $19.9 \%$ & Residual activity & Yes & Yes \\
\hline PpKAIIL-H & No signal & No signal & No signal & $104 \pm 16 \mu \mathrm{M}$ & $273 \pm 47 \mu \mathrm{M}$ & $26.2 \%$ & $69.2 \%$ & Michaelian kinetic & No & No \\
\hline PpKAI2L-L & $43.2^{\circ} \mathrm{C}$ & $44.1^{\circ} \mathrm{C}$ & $44.3^{\circ} \mathrm{C}$ & Not tested & Not tested & $8.3 \%$ & $21.4 \%$ & Residual activity & Yes & Yes \\
\hline AtD 14 & $53.0^{\circ} \mathrm{C}$ & $46.7^{\circ} \mathrm{C}$ & $47.2^{\circ} \mathrm{C}$ & $23 \pm 9 \mu \mathrm{M}$ & $94 \pm 3 \mu \mathrm{M}$ & $100.0 \% 1$ & $100.0 \% 1$ & Single turnover kinetic & Yes $^{2}$ & Yes $^{2}$ \\
\hline
\end{tabular}

a Protein stability assays were performed by nanoDSF. Protein denaturation temperature $\left(T_{m}\right)$ is indicated in the absence (mock) or presence of ligand $((+)$ GR24 and (-)-GR24). A lower temperature (in purple) indicates protein destabilization and a higher temperature (in green) indicates a protein stabilization. A change of protein stability was considered significant when at least $0.5^{\circ} \mathrm{C}$ difference was observed, according to the manufacturer recommendation.

b Affinity of the protein for the different ligands evaluated by intrinsic fluorescence assay.

c PpKAI2L $(5 \mu \mathrm{M})$ enzymatic activity towards (+)-GR24 and (-)-GR24 at $10 \mu \mathrm{M}$, evaluated by calculating the remaining percentage of GR24 isomers in comparison to the amount of ligand incubated in the same condition without protein, after $150 \mathrm{~min}$.

${ }^{d}$ Enzymatic kinetic profile for each PpKAI2L protein (330 nM), evaluated by recording ( \pm )-GC242 (4 $\left.\mu \mathrm{M}\right)$ probe cleavage.

e Covalent PpKAI2L-ligand complexes were investigated by mass spectrometry. "Yes" indicates a detected mass increment of 96.3 Da.

1 For this assay RMS3 was used instead of AtD14.

2 These data are not shown here, but were previously reported (de Saint Germain et al. 2016).

Figure 4. Biochemical characterization of the PpKAI2L proteins.

(A) Chemical structures of the (+)-GR24 and (-)-GR24 enantiomers, and the ( \pm )-GC242 profluorescent probe. (B) Summary of biochemical assays for testing interactions between PpKAI2Ls and SL analogs and probe. 
A

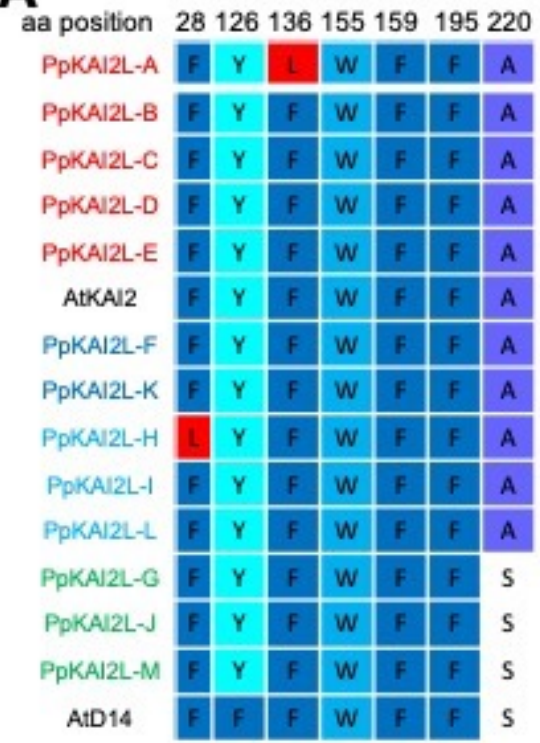

B

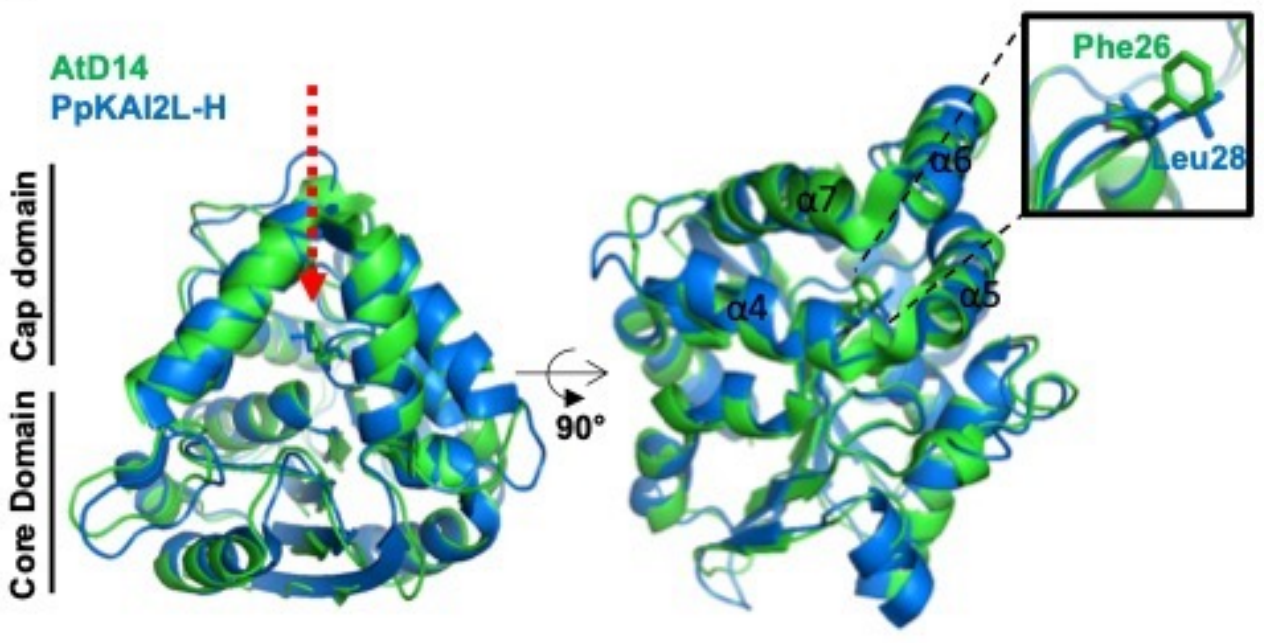

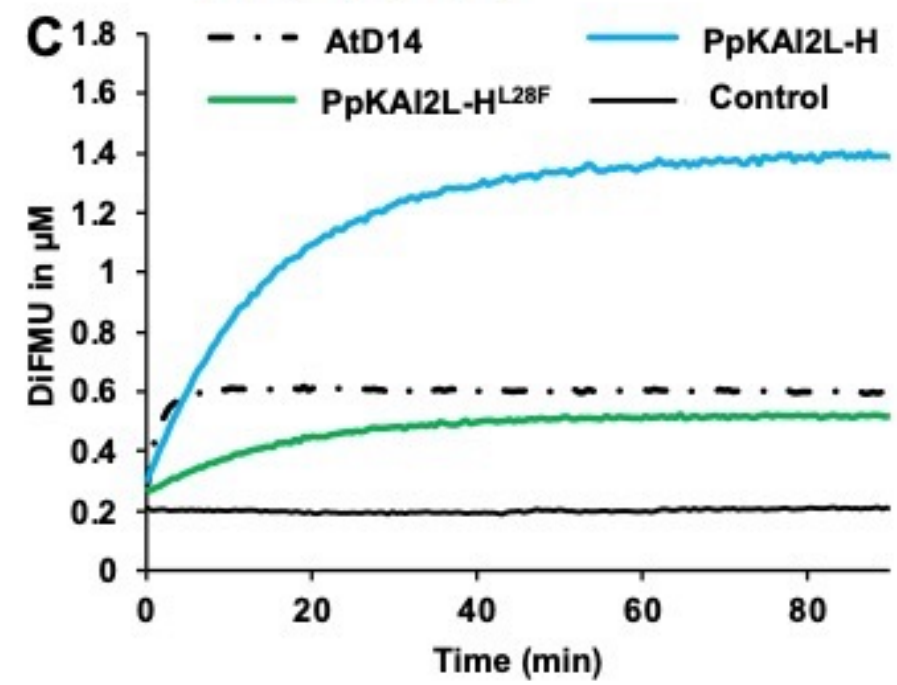

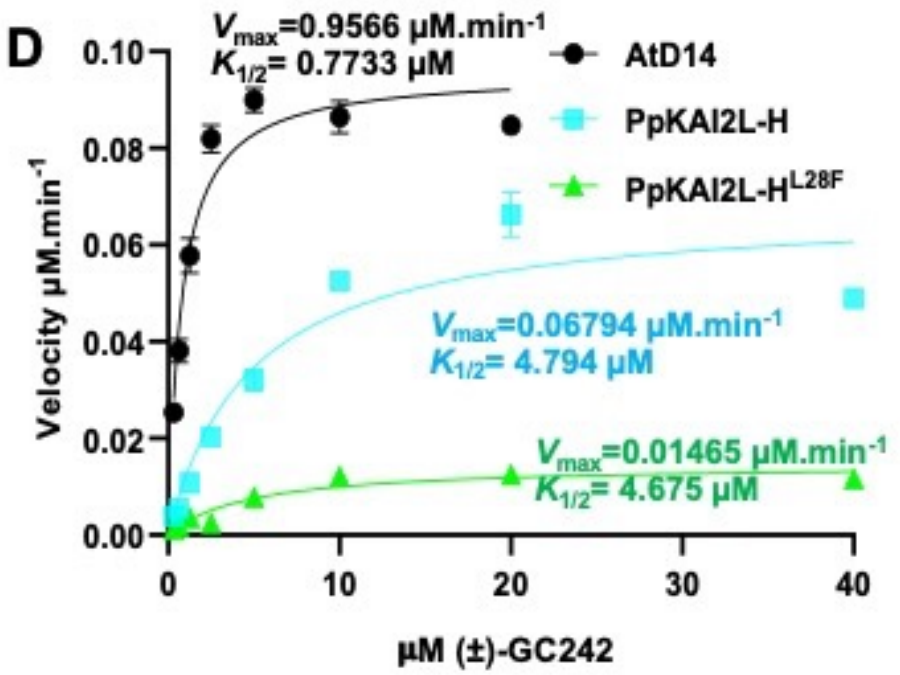

Figure 5. PpKAI2L-H enzymatic activity reveals special feature.

(A) Sequence alignment of active site amino acid residues in PpKAI2L proteins. Amino acids that differ from AtKAI2 are colored in red. A fully expanded alignment can be found in Supplemental Figure S1. (B) Superimposition of the AtD14 and PpKAI2L-H structure showing the position of $\mathrm{Phe}^{28}$ and Leu ${ }^{28}$ residues. Zoom onto helices $\alpha 4$ and $\alpha 5$. (C) Enzyme kinetics for PpKAI2L-H, PpKAI2L-H $\mathrm{H}^{\text {Leu28Phe }}$ and AtD14 incubated with $( \pm)-G C 242$. Progress curves during probe hydrolysis, monitored at $25^{\circ} \mathrm{C}\left(\lambda_{\mathrm{em}} 460 \mathrm{~nm}\right)$. Protein catalyzed hydrolysis with $330 \mathrm{nM}$ of protein and $20 \mu \mathrm{M}$ of probe. These traces represent one of the three replicates and the experiments were repeated twice. (D) Hyperbolic plot of pre-steady state kinetics reaction velocity with $( \pm)$-GC242. Initial velocity was determined with pro-fluorescent probe concentrations from $0.310 \mu \mathrm{M}$ to $40 \mu \mathrm{M}$ and protein at $400 \mathrm{nM}$. Points are the mean of three replicates and error bars represent SE. Experiments were repeated at least three times. 


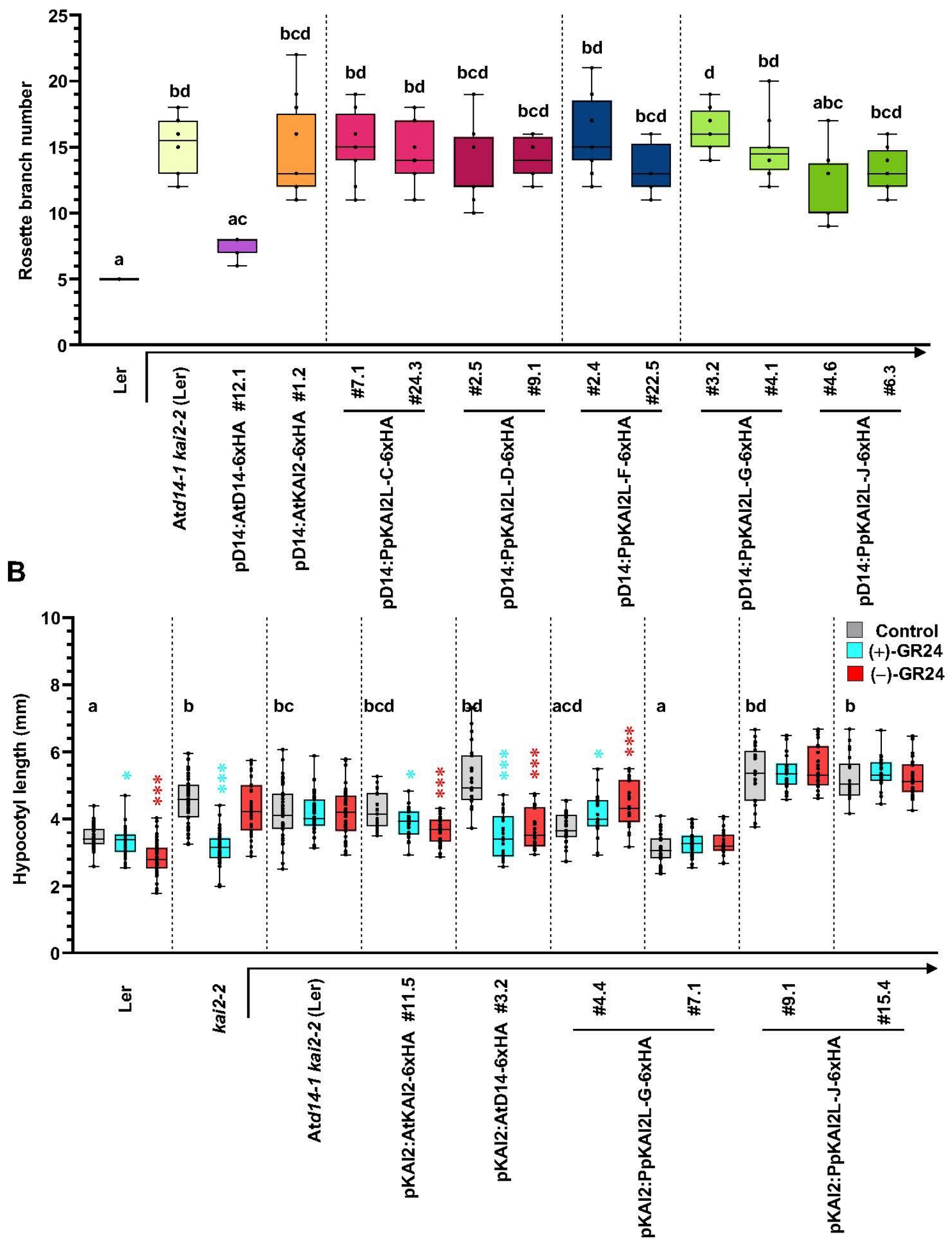

Figure 6. Complementation assays of the Arabidopsis Atd14-1 kai2-2 double mutant with PpKAI2L genes.

Complementation assays of the Atd14-1 kai2-2 mutant (in Ler background), transformed using the AtD14 promoter (A) or AtKAl2 promoter (B) controlling the expression of AtD14, AtKAI2 (controls) or PpKAI2L CDS (as noted below the graph). Ler (WT), kai2-2 and Atd14-1 kai2-2 mutants are shown as controls. (A) Number of rosette axillary branches per plant. Results are mean of $n=12$ plants per genotype, except for Ler and lines pD14:AtD14 \#12.1 and pD14:PpKAI2L-C\#24.3 ( $n=11)$. Different letters indicate significantly different results between genotypes based on a Kruskal-Wallis test $(P<0.05$, Dunn post hoc test). (B) Hypocotyl length under low light, on $1 / 2$ MS medium with DMSO (control, grey bars) $1 \mu \mathrm{M}(+)$-GR24 (turquoise bars) or $1 \mu \mathrm{M}(-)$-GR24 (red bars). Results of $n=20$ to 24 seedlings. Whiskers refer to minimum and maximum values, bars inside the boxplot to the median. Different letters indicate significantly different results between genotypes in control conditions based on a Kruskal-Wallis test $(P<0.05$, Tukey post hoc test). Asterisks in turquoise and red give the statistical significance of response to (+)-GR24 and (-)-GR24 respectively (Mann-Whitney tests, ${ }^{*} 0.01 \leq \mathrm{P}<0.05$; ${ }^{* * *} \mathrm{P} \leq 0.001$ ). 
A
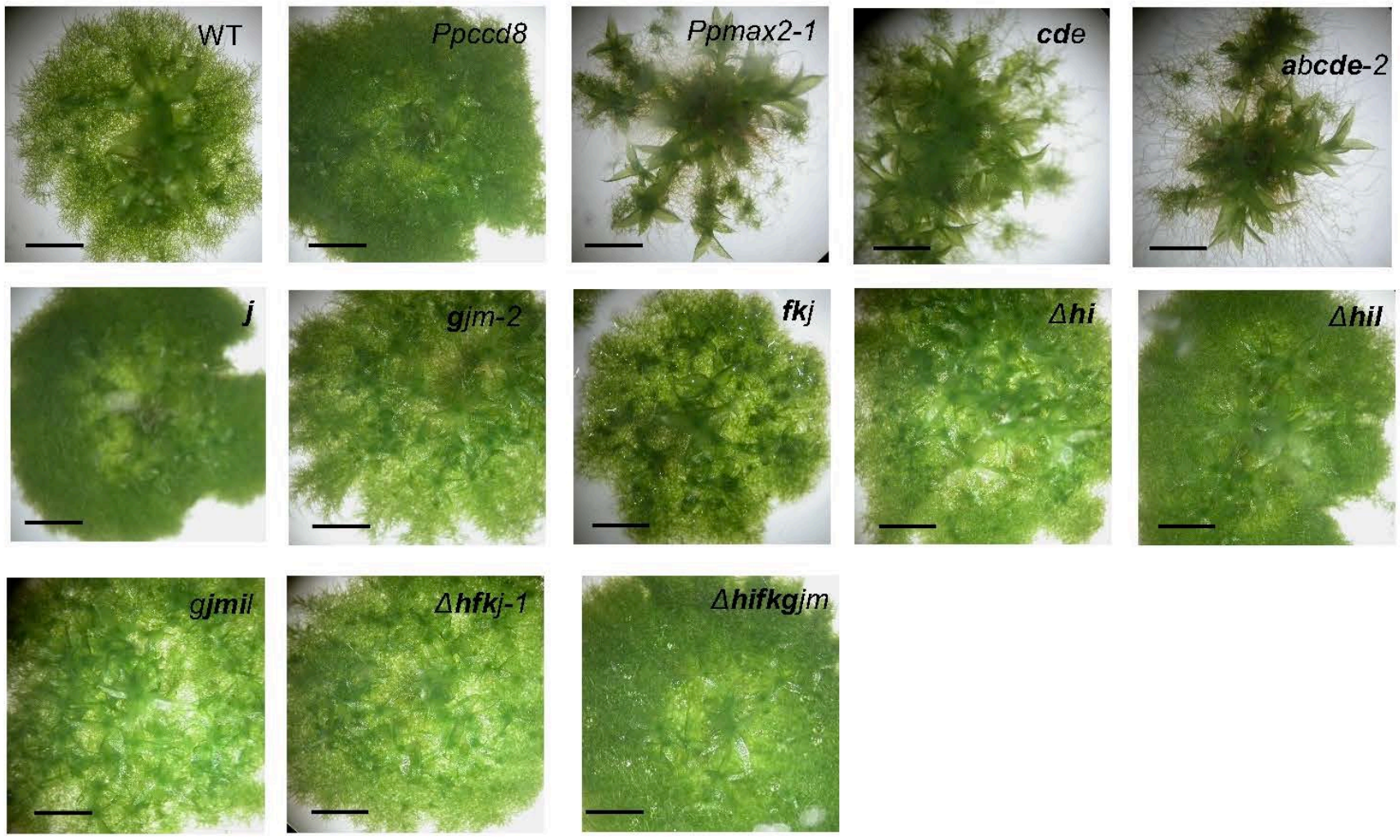

B

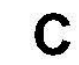

D
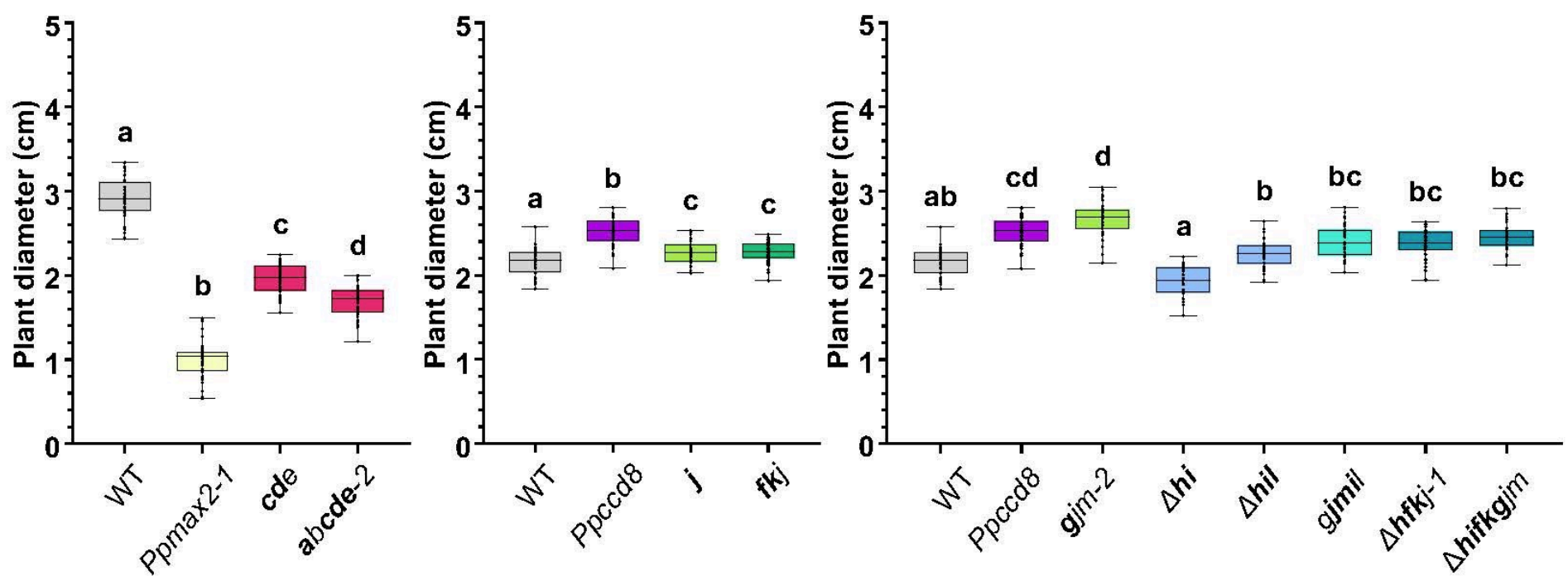

Figure 7. Phenotype of the Ppkai2L mutants in light.

(A) 3-week-old plants. Scale bar = $2 \mathrm{~mm}$ (B) and (C): 30-day-old plants, $(\mathrm{n}>40)$; (D): 28-day-old plants, $(\mathrm{n}=30)$. (B), (C) and (D): All plants were grown on cellophane disks. Whiskers refer to minimum and maximum values, bars inside the boxplot to the median. Letters indicate statistical significance of comparisons between all genotypes based on a Kruskal-Wallis test followed by a Dunn post hoc test $(P<0.05)$. Mutant genotypes carry mutations as described in Supplemental Figure $S 10$ and Supplemental Table S1. Bold letters indicate null mutations. 

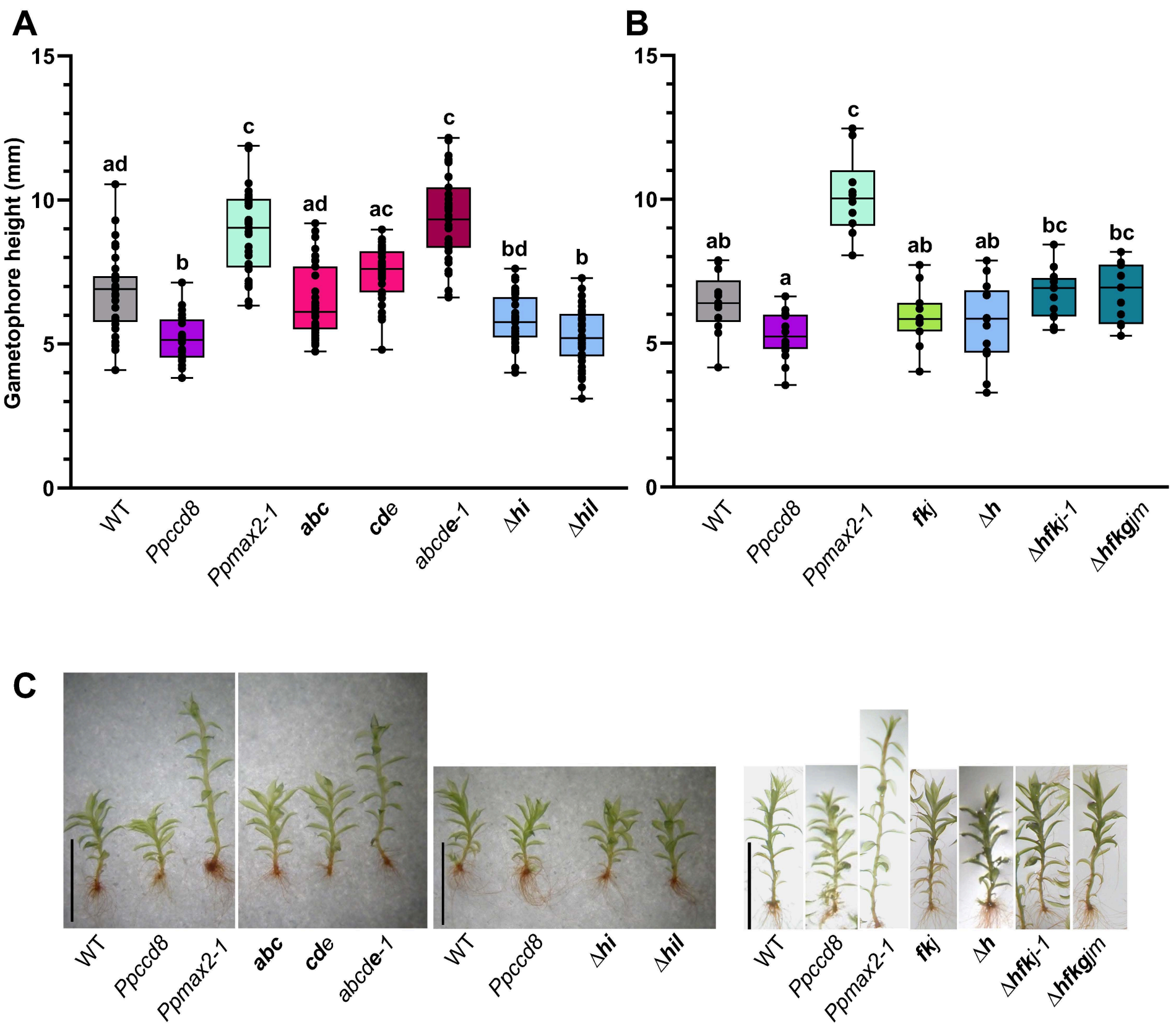

Figure 8. Ppkai2L mutant gametophores in red light.

(A) Gametophore height of Ppkai2L mutants affecting eu-KAI2 clade genes (abc; cde; abcde-1) and clade (HIL) genes ( $\Delta$ hi and $\Delta$ hil), compared to that of WT, Ppccd8 and Ppmax2-1 mutants, following two months growth under red light. Box plots of $\mathrm{n}=32-36$ gametophores, grown in 3 Magenta pots, harboring between 15 and 25 leaves. Whiskers refer to minimum and maximum values, bars inside the boxplot to the median. Letters indicate statistical significance of comparisons between all genotypes based on a Kruskal-Wallis test followed by a Dunn post hoc test $(\mathrm{P}<0.05)$. (B) Gametophore height of Ppkai2L mutants affecting clade (HIL) gene $(\Delta \boldsymbol{h})$, both clades (FK) and (JGM) (fkj) and all three clades (HIL) (FK) and (JGM) ( $\Delta \boldsymbol{h} f \boldsymbol{k} j-1$ and $\Delta \boldsymbol{h}$ fkgjm). Box plots of $\mathrm{n}=11-15$ gametophores, grown in 3 Magenta pots, harboring between 15 and 25 leaves. Whiskers refer to minimum and maximum values, bars inside the boxplot to the median. Letters indicate statistical significance of comparisons between all genotypes based on a Kruskal-Wallis test followed by a Dunn post hoc test $(P<$ 0.05). (C) Examples of gametophores following a 2-month growth under red light, from WT, Ppccd8, Ppmax2-1, and Ppkai2L mutants as shown in (A) and (B). Scale bar $=5 \mathrm{~mm}$. Mutant genotypes carry mutations as described in Supplemental Figure S10 and Supplemental Table S1. Bold letters indicate null mutations. 
A

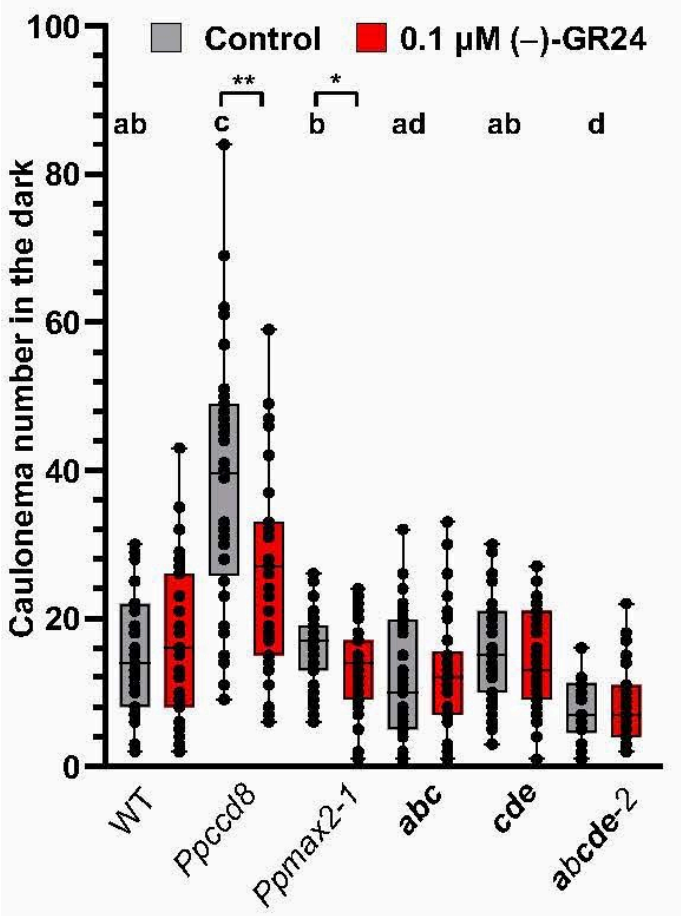

B

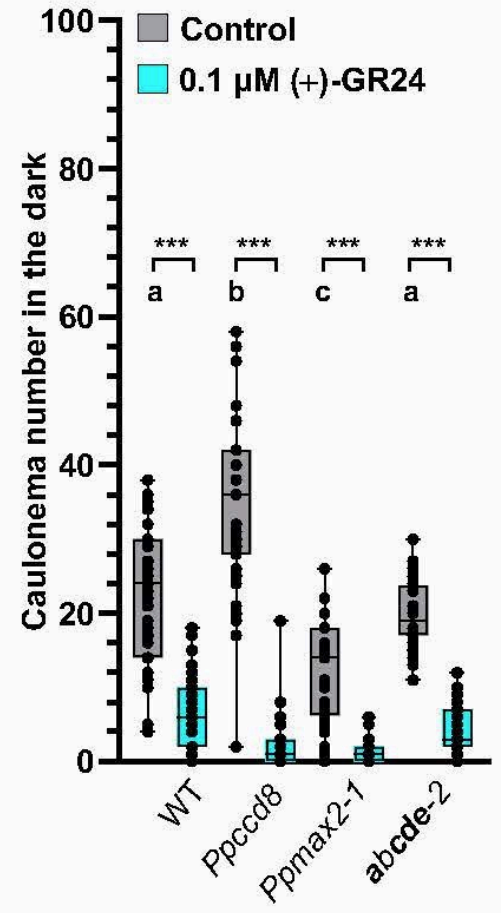

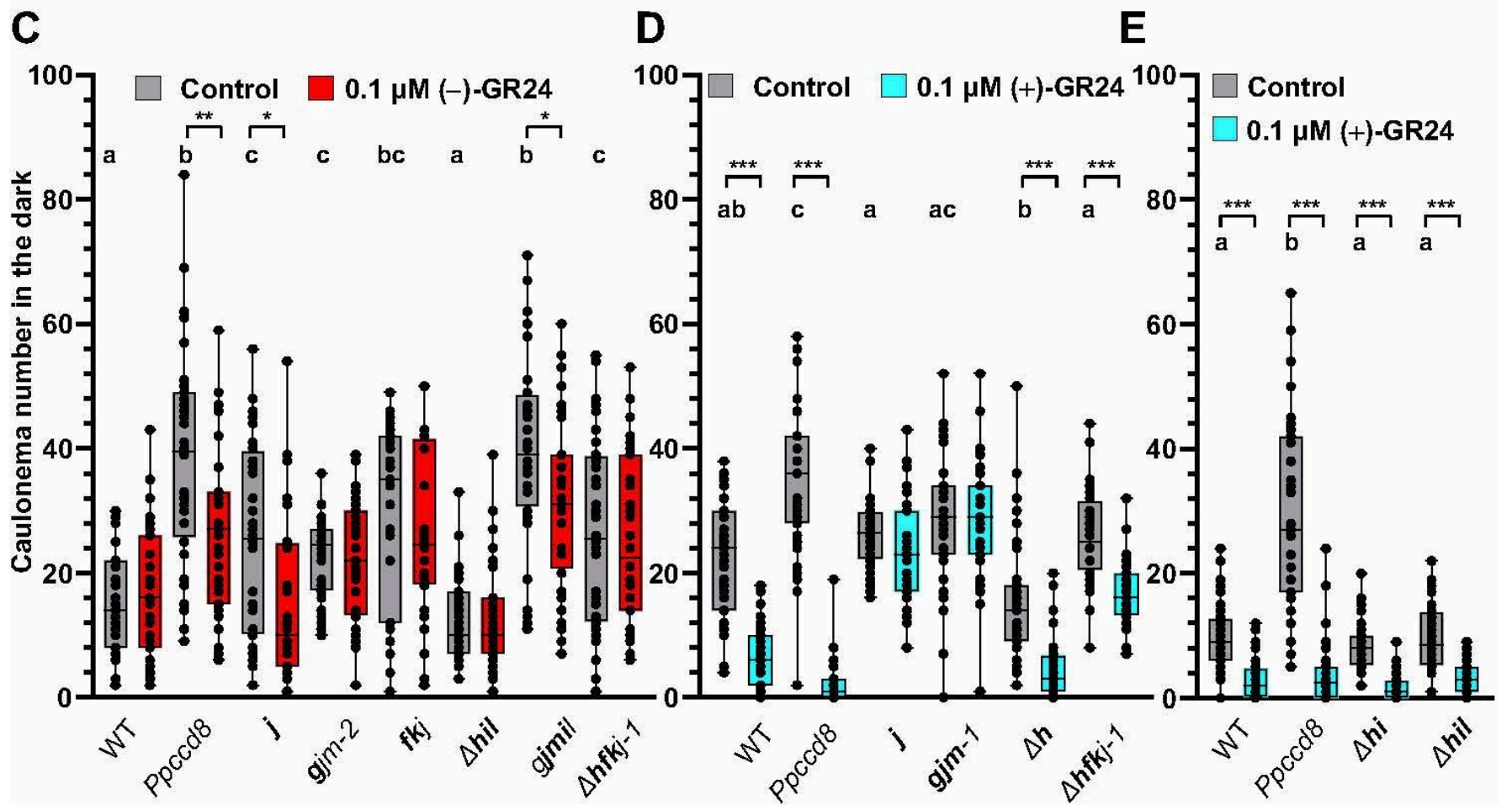

Figure 9. Phenotypic response of Ppkai2L mutants to (-)-GR24 and (+)-GR24 application: caulonema number in the dark.

(A-B) Caulonema numbers from mutants affecting eu-KAI2 clade genes following application of $0.1 \mu \mathrm{M}(-)$-GR24 (in red, A) or $0.1 \mu \mathrm{M}(+)-G R 24$ (in turquoise, B). DMSO was applied as a control treatment (dark grey). WT and both the Ppccd8 and Ppmax21 mutants were used as control genotypes. (C-E) Caulonema numbers from mutants affecting (GJM) clade genes: $\boldsymbol{j}$; gjm-2; gjm1; (FK) and (GJM) clade genes: $\boldsymbol{f k j}$, (HIL) clade genes: $\Delta \boldsymbol{h}, \boldsymbol{\Delta h} \boldsymbol{i}, \Delta \boldsymbol{h i l}$, (HIL) and (GJM) clade genes: gjmil, or all 3 clades genes: $\Delta \boldsymbol{h f k j - 1}$, following application of $0.1 \mu \mathrm{M}(-)$-GR24 (in red, C) or $0.1 \mu \mathrm{M}(+)-G R 24$ (in turquoise, D, E). $0.01 \%$ DMSO was applied as a control treatment (dark grey). WT and the Ppccd8 mutant were used as control genotypes. Mutant genotypes carry mutations described in Supplemental Figure S10 and Supplemental Table S1. Bold letters indicate null mutations. For each genotype/treatment combination, caulonema were counted after two weeks in the dark, from 24 individuals $(n=24)$. Whiskers refer to minimum and maximum values, bars inside the boxplot to the median. Letters indicate statistical significance of comparisons between all genotypes in control conditions, based on a Kruskal-Wallis test followed by a Dunn post hoc test $(P<$ 0.05). Significant differences between control and treated plants within a genotype based on a Kruskal-Wallis test, followed by a Dunn post-hoc test for multiple comparisons: ${ }^{* \star} \mathrm{P}<0.001$; ${ }^{* *} \mathrm{P}<0.01$; ${ }^{*} \mathrm{P}<0.5$. 
PpKUF1LA

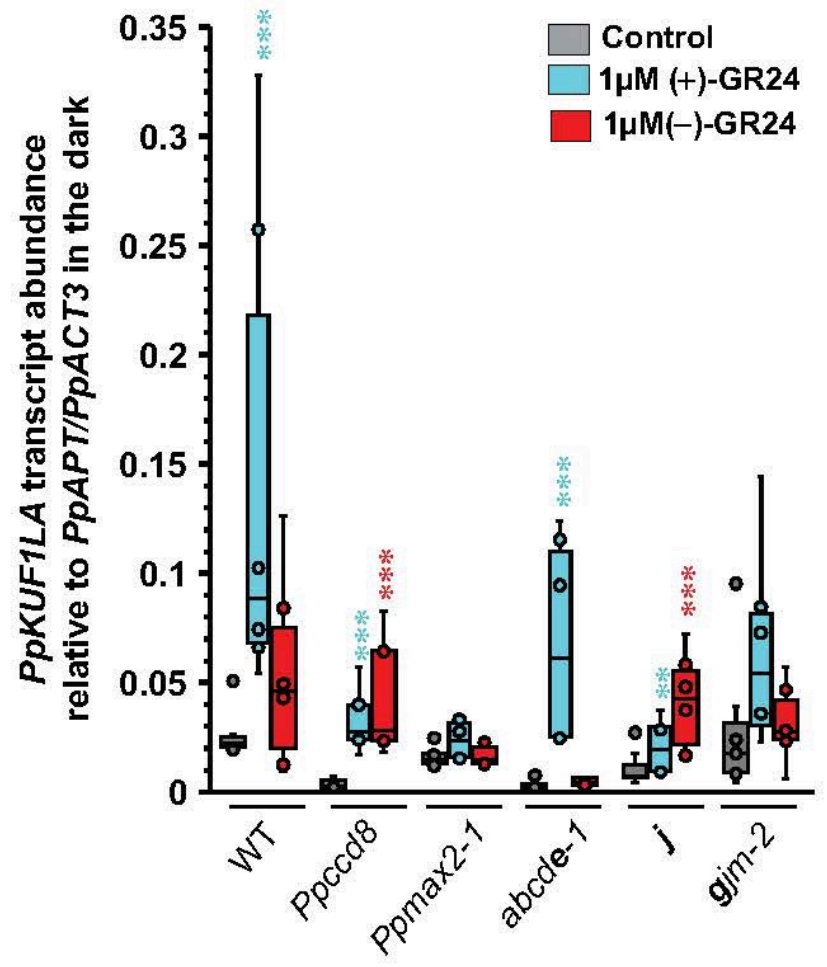

Pp3c6_15020

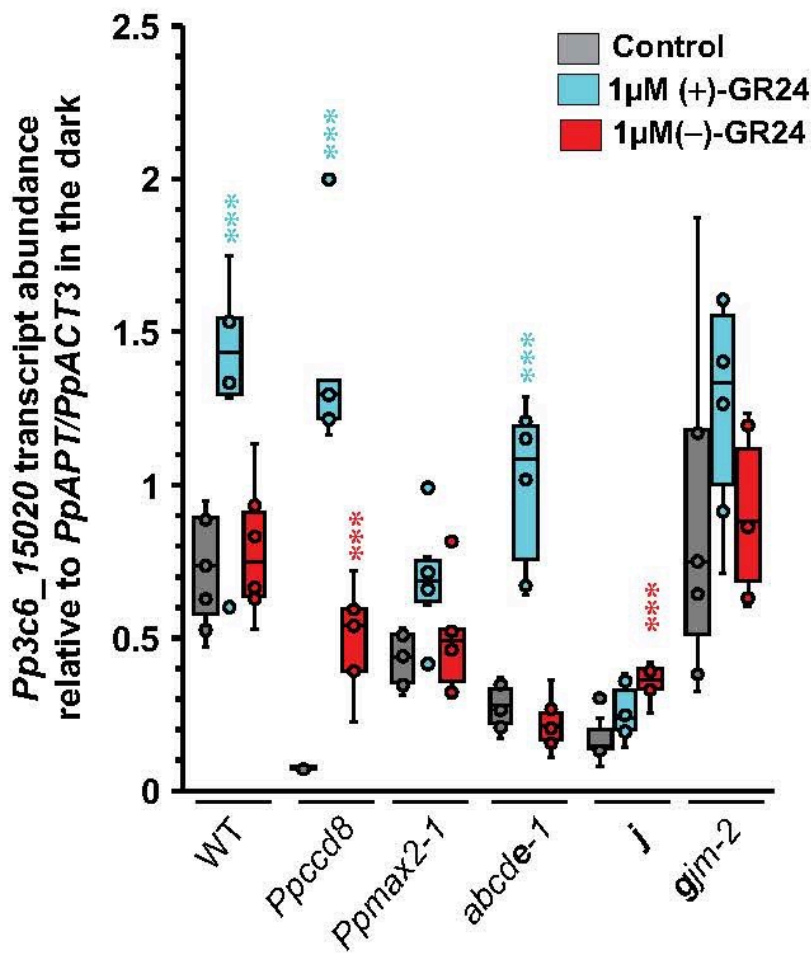

Figure 10. Ppkai2L mutant transcriptional response to (+)-and (-)-GR24.

Transcript abundance analysis of the SL-responsive genes PpKUF1LA and Pp3c6_15020, in WT, Ppccd8, Ppmax2-1 and Ppkai2L mutants abcde-1 (eu-KAl2 clade), $\boldsymbol{j}$ and $\boldsymbol{g j m}$-2 ((GJM) clade), after a 6-hour treatment in the dark with DMSO (control, grey), $1 \mu \mathrm{M}(+)$-GR24 (turquoise) or $1 \mu \mathrm{M}(-)$-GR24 (red). Box plots of at least four biological repeats are shown ( $\geq 4$ ), relative to mean (PpAPT-PpACT3) transcript abundance. Whiskers refer to lower and upper quartiles, bars inside the box to the median. Kruskal-Wallis test followed by a Dunn post hoc test (Asterisks indicate significant differences between the treatment and the corresponding DMSO control ${ }^{* *} \mathrm{P}<0.01$ and $\left.{ }^{* *} \mathrm{P}<0.001\right)$. 


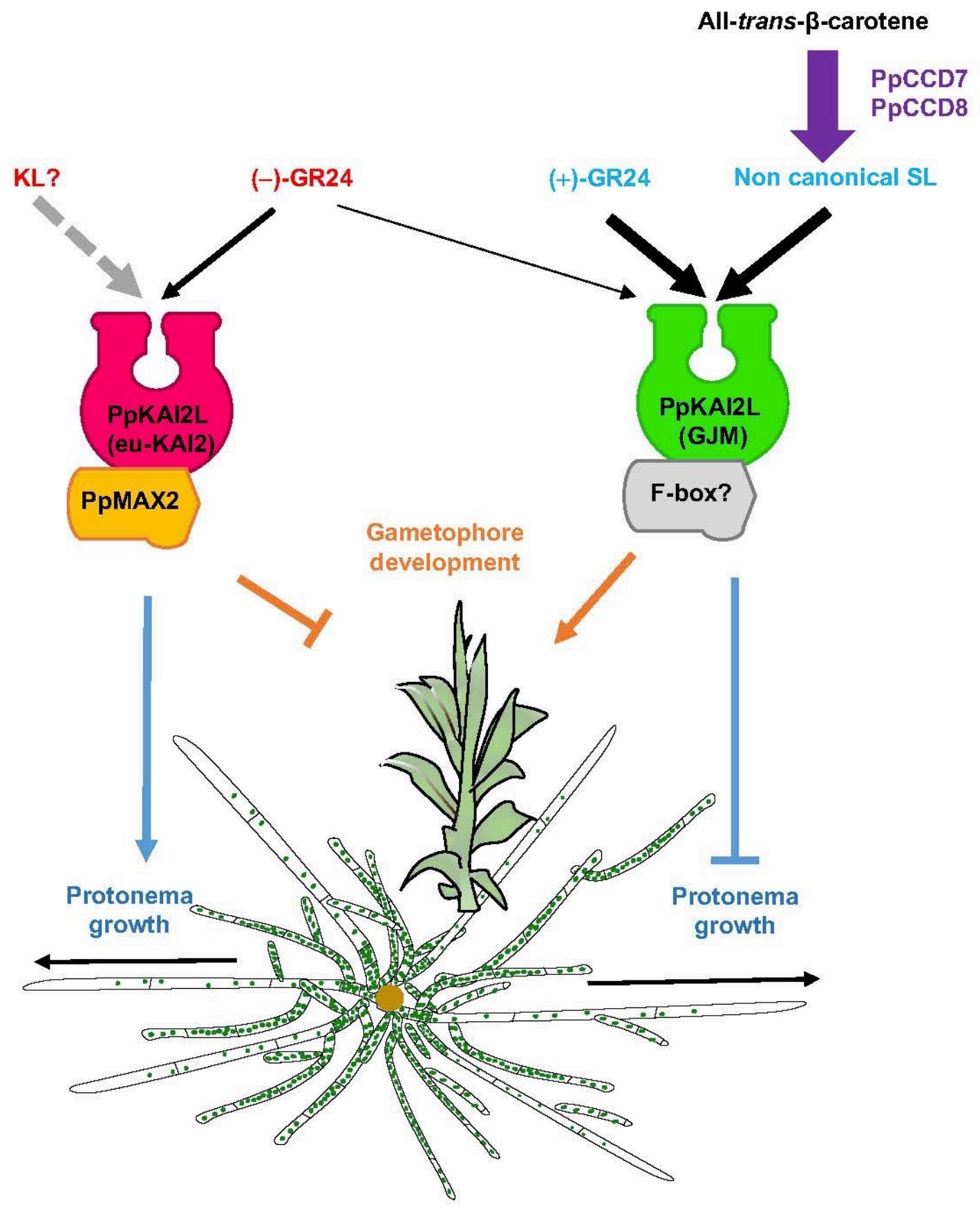

Figure 11. Current model for SL (PpMAX2-independent) and KL (PpMAX2-dependent) perception in $P$. patens.

Eu-KAI2 clade PpKAI2L proteins perceive $\mathrm{KL}$ compounds which promote protonema growth and inhibit gametophore development through a PpMAX2-dependent pathway. (GJM) clade PpKAI2L proteins perceive PpCCD8-derived compounds (noted non-canonical SL) which inhibit protonema growth while promoting gametophore development, in a PpMAX2-independent manner. (+)-GR24 mimics PpCCD8-derived compound effects and is perceived by (GJM) clade PpKAI2L proteins. (-)-GR24 is perceived by both eu-KAI2 clade and (GJM) clade PpKAI2L proteins, making it a poor mimic of moss $\mathrm{KL}$. Grey doted arrow indicates non-demonstrated effect. Thickness of black arrows indicate the strength of the compound's effect. 